\title{
Biological Activity and In Silico Study of 3-Modified Derivatives of Betulin and Betulinic Aldehyde
}

\author{
Ewa Bębenek ${ }^{1, *}$, Elwira Chrobak ${ }^{1}$, Krzysztof Marciniec ${ }^{1}$, Monika Kadela-Tomanek ${ }^{1}$, \\ Justyna Trynda ${ }^{2}$, Joanna Wietrzyk ${ }^{3}$ and Stanisław Boryczka ${ }^{1}$ \\ 1 Medical University of Silesia in Katowice, School of Pharmacy with the Division of Laboratory Medicine in \\ Sosnowiec, Department of Organic Chemistry, 4 Jagiellońska Str., 41-200 Sosnowiec, Poland; \\ echrobak@sum.edu.pl (E.C.); kmarciniec@sum.edu.pl (K.M.); mkadela@sum.edu.pl (M.K.-T.); \\ boryczka@sum.edu.pl (S.B.) \\ 2 Wroclaw University of Environmental and Life Science, Department of Experimental Biology, \\ 27b Norwida Str., 50-375 Wrocław, Poland; justyna.trynda@upwr.edu.pl \\ 3 Polish Academy of Sciences, Ludwik Hirszfeld Institute of Immunology and Experimental Therapy, \\ Department of Experimental Oncology, 12 Rudolfa Weigla Str., 53-114 Wrocław, Poland; \\ wietrzyk@iitd.pan.wroc.pl \\ * Correspondence: ebebenek@sum.edu.pl; Tel.: +48-323-641-666
}

Received: 15 February 2019; Accepted: 12 March 2019; Published: 19 March 2019

\begin{abstract}
A series of 3-substituted derivatives of betulin and betulinic aldehyde were synthesized as promising anticancer agents. The newly triterpenes were tested against five human cancer cell lines like biphenotypic B myelomonocytic leukaemia (MV-4-11), adenocarcinoma (A549), prostate (Du-145), melanoma (Hs294T), breast adenocarcinoma (MCF-7) and normal human mammary gland (MCF-10A). The compound 9 showed towards Du-145, MCF-7 and Hs294T cells significant antiproliferative activity with $\mathrm{IC}_{50}$ ranging from 7.3 to $10.6 \mu \mathrm{M}$. The evaluation of ADME properties of all compounds also includes their pharmacokinetic profile. The calculated TPSA values for synthetized derivatives are in the range between $43.38 \AA^{2}$ and $55.77 \AA^{2}$ suggesting high oral bioavailability. The molecular docking calculations showed that triterpene 9 fits the active site of the serine/threonine protein kinase Akt.
\end{abstract}

Keywords: betulin; betulinic aldehyde; antiproliferative activity; ADME; molecular docking

\section{Introduction}

Presently, more than 120 drugs of plant origin are used for the treatment or amelioration of various diseases. Many promising candidates for medicines derived from natural sources have some undesirable properties, including poor water solubility and low thermal stability. However, these substances can be used as lead compounds for the preparation of new drugs [1].

The large class of natural compounds are triterpenes, the secondary plant metabolites, represented by betulin 1 . The outer bark layer of the birch tree contain as major compound betulin 1 (Figure 1) (12-30\% of dry weight) and trace amounts of other triterpenes such as allobetulin, betulinic acid, betulinic aldehyde and lupeol [2]. The biological properties reported for betulin $\mathbf{1}$ and its derivatives include anticancer [3,4], antiviral [5,6], antibacterial [7,8] and anti-inflammatory activity [9-11]. 


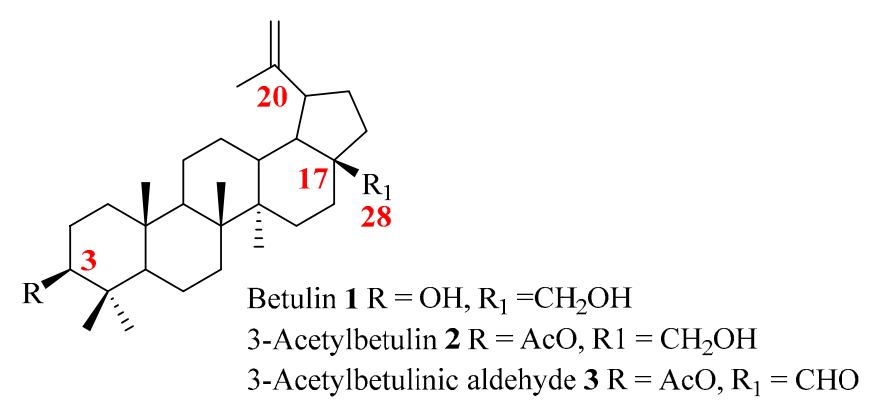

Figure 1. Chemical structure of triterpenes 1-3.

The structural diversity of triterpenes are obtained generally through chemical modification of their functional groups at the C-3, C-28, C-20 and C-17 positions. The promising semisynthetic derivatives of triterpenes as anticancer agents were obtained by introduction various functional groups at the C-3 position. An example of such substances are 3-methyl- and 3-ethyl-phthalates of betulinic acid showed significant cytotoxicity compared to betulinic acid towards six tested cancer cell lines. The most active compound was 3-methyl-phthalate exhibited cytotoxic activity towards cancer cells from different histogenetic origins like epithelial tumours (PC-3, HT-29), neuroectodermal tumours (SK-MEL2) and mesenchymal tumours (K562, K562-tax, CEM) [12]. Received by [13] 3-glutaryl-, 3-succinyl- and 3-acetylbetulinic acids exhibited higher activity than the betulinic acid $\left(\mathrm{IC}_{50}=8.4 \mu \mathrm{g} / \mathrm{mL}\right)$ against the lung $\mathrm{A} 549$ cell line with $\mathrm{IC}_{50}$ ranging from 6.4 to $7.4 \mu \mathrm{g} / \mathrm{mL}$.

Moreover, it was observed that the derivatives of betulinic acid bearing a shorter alkyl chain on the acyl group at the C-3 position were more toxic on the A549 cells [13]. Cytotoxic data of the 3-modified derivatives of dihydrobetulinic acid indicates that presence of the 4-fluorophenyl-hydrazono moiety at the C-3 position led to compound with significant cytotoxic activity towards cells of leukaemia (MOLT-4), ovary (PA-1) and colon (HT-29) cancer. Additionally, it appeared, that derivative containing the 4-fluorophenyl-hydrazono group at the C-3 position had better water solubility $(124.7 \mu \mathrm{M})$ as compared to betulinic acid $(<1 \mu \mathrm{M})$ [14]. The introduction the triazolyl moiety at the C-3 position of betulin 1 and 2'-methylimidazolyl moiety at the C-3 position of betulinic acid produce a novel derivatives of triterpenes with potent anticancer activity. The cytotoxic activity of 3-substituted tiazole and imidazole derivatives was evaluated towards different tumours type such as prostate adenocarcinoma (PC-3), leukaemia (Jurkat), cervical adenocarcinoma (HeLa), hepatocellular carcinoma (HepG2) and colon adenocarcinoma (HT-29). These compounds showed a better cytotoxic profile than the betulinic acid $\left(\mathrm{IC}_{50}=12.8-36.4 \mu \mathrm{M}\right)$ with $\mathrm{IC}_{50}$ values ranging from 2.2 to $12.2 \mu \mathrm{M}$ in the tested cancer cell lines [15].

The addition of polar group at the C-3 position of betulinic acid such as $\alpha$-L-rhamnopyranose moiety increase the water solubility and hence the antiproliferative activity against two applied human cancer cell lines A549 (lung carcinoma) and DLD-1 (colon adenocarcinoma) [16]. Cui et al. synthesized a new 3-substituted derivatives of betulinic acid by introducing nitrogen-containing heterocycles connected to the triterpene skeleton with an amide linkage. The all 3-amide derivatives of betulinic acid were tested for their in vitro antiproliferative activity towards eight human cell lines like prostate cancer (Du-145, PC-3), colon carcinoma (HT-29, HCT-116), breast cancer (T47D, MCF-7, MDA-MB-231) and multidrug-resistant breast cancer (MCF-7/ADR). Especially, derivative of betulinic acid containing the 4-piperidinecarboxamide group at the $\mathrm{C}-3$ position had the significant cytotoxic activity $\left(\mathrm{IC}_{50}\right.$ $=0.33 \mu \mathrm{M})$ towards MCF-7/ADR cells, about 117-fold more potent than the betulinic acid $\left(\mathrm{IC}_{50}=\right.$ $38.5 \mu \mathrm{M}$ ). It was demonstrated, that this compound induced apoptosis on the MCF-7 and MCF-7/ADR cell lines and exhibited potent antimetastatic activity on the MDA-MB-231 cells [17].

Based on the above information and our previous studies we synthesized the newly 3-substituted derivatives of betulin and betulinic aldehyde with alkyl, alkenyl and alkynyl chain on the acyl group [18-21]. The antiproliferative activity in vitro was evaluated towards five human cancer cell lines like biphenotypic B myelomonocytic leukaemia (MV-4-11), adenocarcinoma (A549), prostate (Du-145), 
melanoma (Hs294T), breast adenocarcinoma (MCF-7) and normal human mammary gland (MCF-10A) by the MTT and the SRB methods. The predicted ADME properties were used to determinate of bioavailability of newly 3-substituted triterpenes. Additionally, the molecular docking studies were conducted to identify the binding mechanism of obtained compounds with the serine/threonine kinase Akt.

\section{Results and Discussion}

\subsection{Chemistry}

Firstly, the naturally occurring betulin $\mathbf{1}$ was used as starting material to the synthesis of 3-acetylbetulin 2 and 3-acetylbetulinic aldehyde 3 (Figure 1). The synthesis of compounds 2-3 was carried out on the basis of the procedure described by Liu et al. [22].

As shown in Scheme 1, the monoprotection of betulin 1 by pyridinium p-toluenesulfonate (PPTS) and dihydropyran (DHP) in dichloromethane gave the tetrahydropyranyl ether 4 in $57 \%$ yield $[23,24]$. The series of 3-substituted betulin derivatives 5-14 was obtained in the two-step synthesis involving the esterification reaction of the secondary hydroxyl group at the $\mathrm{C}-3$ position followed by the deprotection of the THP group with PPTS in ethanol.

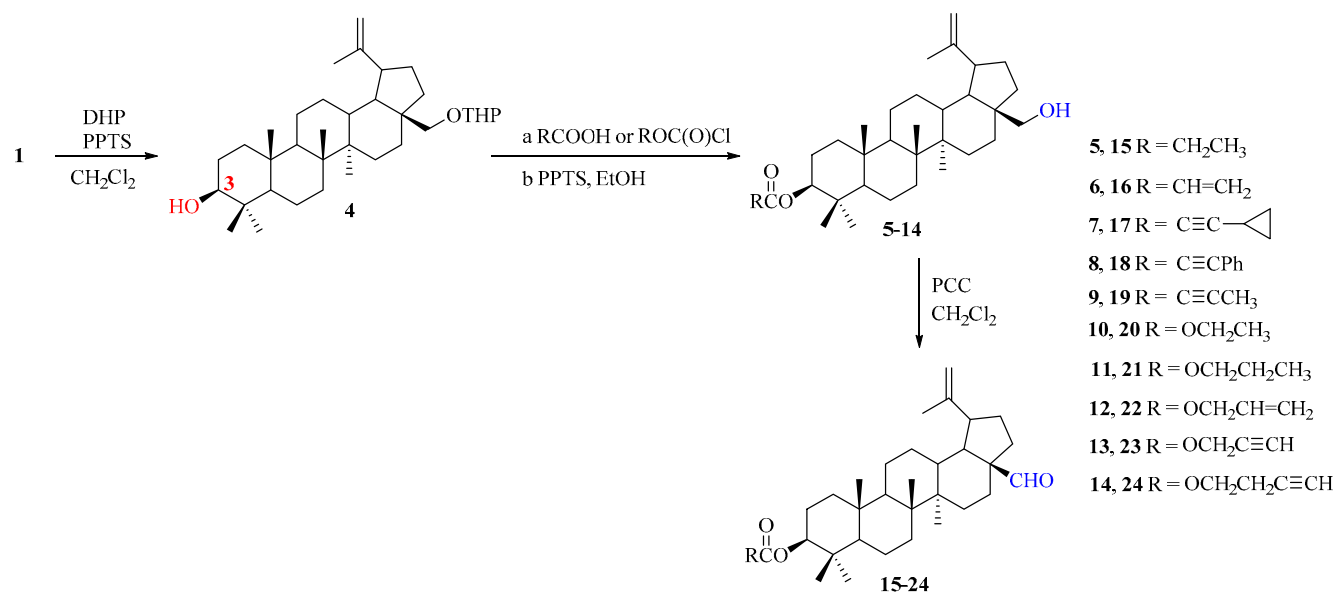

Scheme 1. Synthesis of the 3-modified derivatives of betulin and betulinic aldehyde 5-24.

The resulting esters 5-14 were oxidized by pyridinium chlorochromate (PCC) in dry dichloromethane to the derivatives of betulinic aldehyde $\mathbf{1 5 - 2 4}$ in $61-84 \%$ yields. The chemical structures of all obtained triterpenes 5-24 were confirmed by ${ }^{1} \mathrm{H}$ NMR, ${ }^{13} \mathrm{C}$ NMR, IR and HR-MS techniques (Supplementary data, Figures S1-S40).

\subsection{Antiproliferative Activity}

In the first stage of the in vitro antiproliferative activity research the screening test of the compounds 5-24 towards the human biphenotypic B myelomonocytic leukaemia (MV-4-11) cell line was performed. Betulin 1, 3-acetylbetulin 2, 3-acetylbetulinic aldehyde 3 and cisplatin were applied as the reference compounds. The results of cytotoxicity of the derivatives 5-24 towards the MV-4-11 cells are reported in Table 1 as $\mathrm{IC}_{50}(\mu \mathrm{M})$. It has been observed, that presence of an alkenyl and alkynyl moiety at the C-3 position of betulin $\mathbf{1}$ molecule moderately increased cytotoxicity towards MV-4-11 cells. The rank order of the anticancer activity against MV-4-11 is as follows: $6>\mathbf{9}>\mathbf{1 6}>\mathbf{1 3}>$ $14>10>12>20>24>23>11>19>22>5>7>15>21>17>8=18$.

The compound 6 having 2-propenoyl group showed the highest anticancer activity with $\mathrm{IC}_{50}$ value of $4.2 \mu \mathrm{M}$. The 3-alkynyl derivatives 9 and 13-14 exhibited more potent cytotoxicity than 3-acetylbetulin 2 with $\mathrm{IC}_{50}$ values ranging from 4.5 to $8.9 \mu \mathrm{M}$. The remaining compounds showed moderate activity towards MV-4-11 with $\mathrm{IC}_{50}$ value above $10 \mu \mathrm{M}$. It should be noted, that the presence 
of the formyl group at the C-17 position of compounds 15-24, resulted in decrease of activity towards MV-4-11 cells compared to their 3-substituted analogues 5-14. In the case of derivatives of betulinic aldehyde, only compound 16 exhibited significant activity against MV-4-11 cells, 6-fold more potent than the 3-acetylbetulinic aldehyde 3.

Table 1. In vitro antiproliferative activity of triterpenes 5-24, betulin 1, 3-acetylbetulin 2, 3acetylbetulinic aldehyde 3 and cisplatin towards the human biphenotypic B myelomonocytic leukaemia (MV-4-11) cell line.

\begin{tabular}{cccc}
\hline Compound & $\begin{array}{c}\text { MV-4-11 } \\
\mathbf{I C}_{\mathbf{5 0}}(\boldsymbol{\mu M})\end{array}$ & Compound & $\begin{array}{c}\text { MV-4-11 } \\
\mathbf{I C}_{\mathbf{5 0}}(\boldsymbol{\mu M})\end{array}$ \\
\hline $\mathbf{5}$ & $38.9 \pm 11.8$ & $\mathbf{1 5}$ & $71.8 \pm 22.9$ \\
$\mathbf{6}$ & $4.2 \pm 1.2$ & $\mathbf{1 6}$ & $5.8 \pm 1.8$ \\
$\mathbf{7}$ & $62.4 \pm 9.9$ & $\mathbf{1 7}$ & $86.7 \pm 42.8$ \\
$\mathbf{8}$ & $\mathrm{Neg}$ & $\mathbf{1 8}$ & $\mathrm{Neg}$ \\
$\mathbf{9}$ & $4.5 \pm 1.7$ & $\mathbf{1 9}$ & $35.9 \pm 4.9$ \\
$\mathbf{1 0}$ & $20.0 \pm 1.5$ & $\mathbf{2 0}$ & $25.1 \pm 7.2$ \\
$\mathbf{1 1}$ & $30.8 \pm 1.5$ & $\mathbf{2 1}$ & $80.2 \pm 14.4$ \\
$\mathbf{1 2}$ & $20.1 \pm 4.7$ & $\mathbf{2 2}$ & $38.3 \pm 4.6$ \\
$\mathbf{1 3}$ & $7.4 \pm 1.7$ & $\mathbf{2 3}$ & $30.0 \pm 6.1$ \\
$\mathbf{1 4}$ & $8.9 \pm 0.9$ & $\mathbf{2 4}$ & $28.3 \pm 7.4$ \\
\hline Betulin $\mathbf{1}$ & $8.1 \pm 2.9$ & & \\
$\mathbf{2}$ & $27.2 \pm 5.9$ & \multicolumn{3}{c}{} \\
$\mathbf{3}$ & $35.0 \pm 12.8$ & \\
Cisplatin & $1.0 \pm 0.27$ & \\
\multicolumn{5}{c}{ Neg-negative in the concentration used. }
\end{tabular}

Among the tested compounds only those with level of the anticancer activity which was close or higher to betulin 1, were selected to the second stage of the study. The compounds 6, 9, 13-14, 16, betulin 1 and cisplatin were evaluated in vitro for antiproliferative activity against human cell lines like adenocarcinoma (A549), prostate (Du-145), melanoma (Hs294T), breast adenocarcinoma (MCF-7) and normal mammary gland (MCF-10A). The results of antiproliferative activity of the triterpenes 6, 9, 13-14, 16 against five applied cell lines (A549, Du-145, Hs294T, MCF-7 and MCF-10A) are presented in Table 2.

Table 2. In vitro antiproliferative activity of triterpenes 6, 9, 13-14, 16, betulin 1 and cisplatin towards the human cancer cell lines: adenocarcinoma (A549), prostate (Du-145), melanoma (Hs294T), breast adenocarcinoma (MCF-7) and normal mammary gland (MCF-10A).

\begin{tabular}{|c|c|c|c|c|c|}
\hline \multirow{2}{*}{ Compound } & \multicolumn{5}{|c|}{ Antiproliferative Activity $\mathrm{IC}_{50}(\mu \mathrm{M})$} \\
\hline & A549 & Du-145 & Hs294T & MCF-7 & MCF-10A \\
\hline 6 & $23.3 \pm 1.8$ & $84.5 \pm 39.4$ & $69.0 \pm 3,4$ & $45.9 \pm 3.8$ & $119.8 \pm 15.3$ \\
\hline 9 & $83.5 \pm 25.3$ & $7.3 \pm 0.8$ & $10.6 \pm 4.3$ & $10.4 \pm 5.7$ & $71.7 \pm 8.6$ \\
\hline 13 & $14.9 \pm 2.5$ & $95.1 \pm 16.8$ & $82.7 \pm 8.4$ & $28.9 \pm 4.6$ & Neg \\
\hline 14 & $33.8 \pm 2.8$ & $126.1 \pm 13.2$ & $164.0 \pm 15.8$ & $39.2 \pm 6.9$ & Neg \\
\hline 16 & $15.1 \pm 5.0$ & $67.1 \pm 4.6$ & $74.4 \pm 7.3$ & $60.4 \pm 4.4$ & $112.6 \pm 11.7$ \\
\hline Betulin 1 & $8.6 \pm 0.7$ & $44.0 \pm 19.4$ & $44.1 \pm 7.2$ & $26.2 \pm 6.5$ & $124.2 \pm 41.6$ \\
\hline Cisplatin & $4.3 \pm 1.3$ & $1.6 \pm 0.3$ & $6.3 \pm 1.3$ & $7.7 \pm 0.3$ & $14.7 \pm 1.0$ \\
\hline
\end{tabular}

Our studies showed moderate activity of derivatives 6, 13-14, 16 against the tested cell lines. The most active compound was derivative 9 for which the $\mathrm{IC}_{50}$ values are in the range $7.3-83.5 \mu \mathrm{M}$. The introduction of the 2-butynoyl group at the C-3 position in compound 9 resulted in an increase of antiproliferative activity in Du-145, Hs294T and MCF-7 cell lines when compared with betulin 1. Figure 2 present the Selectivity Index (SI) calculated for compounds 6, 9, 16 and betulin 1 according 
to the formula: $\mathrm{SI}=\mathrm{IC}_{50}$ for normal mammary gland $/ \mathrm{IC}_{50}$ on corresponding tumour cell line. Additionally, triterpene 9 was more selective towards Du-145 (SI=9.82), Hs294T (SI = 6.76) and MCF-7 $(\mathrm{SI}=6.89)$ cells than the reference compound 1 . The obtained results exhibited that carbon-carbon triple bond of carboxyl moiety at the C-3 position is favourable for the antiproliferative activity.

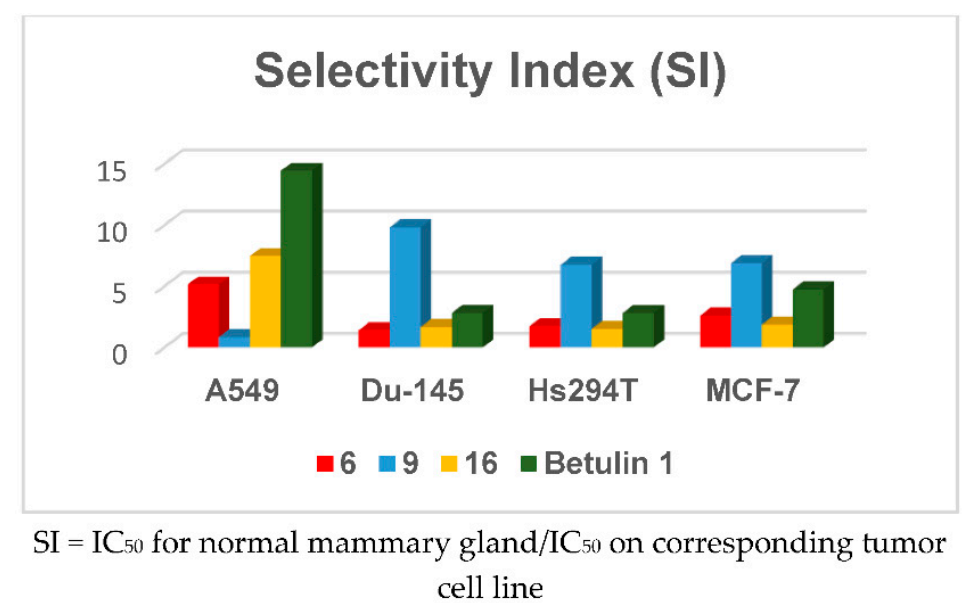

Figure 2. Selectivity Index (SI) of the triterpenes 6, 9, 16 and betulin 1 towards tumour cells.

\subsection{In Silico Study}

Lipinski's "Rule of five" and Veber's criteria are used to assess the similarity of a compound to a drug substance [25-27]. This is related to the determination of such properties as blood (plasma)-brain partition coefficient permeant $(\log B B B)$, lipophilicity $(\log P)$, number of hydrogen bond acceptors and donors (HBA and HBD) and topological polar surface area (TPSA). The ADME parameters of the newly synthesized triterpenes are detailed in Table 3.

The derivatives 5-24 demonstrate the octanol water partition coefficient $(\log \mathrm{P})$ in the range 7.67-9.72, which may be evidence of low ability of compounds to cross the cell membranes. As can be seen in the Table 3, the HBA and HBD values of the 3-substituted derivatives are within the preferred range (HBA $\leq 10$ and $\mathrm{HBD} \leq 5)$. The number of hydrogen bond acceptors (HBA) and hydrogen bond donors (HBD) of the compounds 5-24 are in the range $0-4$.

The TPSA values for all compounds are found in the range between $43.38 \AA^{2}$ and $55.77 \AA^{2}$, which indicates their high oral bioavailability. In addition, low values TPSA of less than $60 \AA^{2}$ shows good permeability through the Blood-Brain Barrier (BBB). The all triterpenes 5-24 exhibit the $\log B B>-1$, which suggest their good transport to the brain. The results of in silico study shows, that these compounds can be considered as promising candidates for the treatment of the central nervous system (CNS).

Table 3. In silico calculation of selected ADME parameters of triterpenes 5-24.

\begin{tabular}{cccccc}
\hline Compounds & LogBB $^{\mathbf{a}}$ & $\mathbf{c L o g}^{\mathbf{b}}$ & HBA $^{\mathbf{c}}$ & HBD $^{\mathbf{d}}$ & TPSA $^{\mathbf{e}}$ \\
\hline $\mathbf{5}$ & 0.3 & 8.42 & 3 & 1 & 46.53 \\
$\mathbf{6}$ & 0 & 8.23 & 3 & 1 & 46.53 \\
$\mathbf{7}$ & -0.42 & 8.85 & 3 & 1 & 46.53 \\
$\mathbf{8}$ & -0.79 & 9.72 & 3 & 1 & 46.53 \\
$\mathbf{9}$ & -0.3 & 8.31 & 3 & 1 & 46.53 \\
$\mathbf{1 0}$ & 0.07 & 7.94 & 4 & 1 & 55.77 \\
$\mathbf{1 1}$ & 0.01 & 8.39 & 4 & 1 & 55.77 \\
$\mathbf{1 2}$ & 0.03 & 8.01 & 4 & 1 & 55.77 \\
$\mathbf{1 3}$ & -0.17 & 7.67 & 4 & 1 & 55.77 \\
$\mathbf{1 4}$ & -0.24 & 8.01 & 4 & 1 & 55.77 \\
$\mathbf{1 5}$ & 0.04 & 8.37 & 3 & 0 & 43.38 \\
$\mathbf{1 6}$ & 0.14 & 8.16 & 3 & 0 & 43.38 \\
\hline
\end{tabular}


Table 3. Cont.

\begin{tabular}{cccccc}
\hline Compounds & LogBB $^{\mathbf{a}}$ & $\mathbf{c L o g P}^{\mathbf{b}}$ & HBA $^{\mathbf{c}}$ & HBD $^{\mathbf{d}}$ & TPSA $^{\mathbf{e}}$ \\
\hline $\mathbf{1 7}$ & -0.21 & 8.76 & 3 & 0 & 43.38 \\
$\mathbf{1 8}$ & -0.7 & 9.56 & 3 & 0 & 43.38 \\
$\mathbf{1 9}$ & -0.1 & 8.32 & 3 & 0 & 43.38 \\
$\mathbf{2 0}$ & 0.07 & 7.99 & 4 & 0 & 52.61 \\
$\mathbf{2 1}$ & 0.03 & 8.26 & 4 & 0 & 52.61 \\
$\mathbf{2 2}$ & 0.07 & 8.17 & 4 & 0 & 52.61 \\
$\mathbf{2 3}$ & -0.11 & 7.79 & 4 & 0 & 52.61 \\
$\mathbf{2 4}$ & -0.15 & 8.12 & 4 & 0 & 52.61 \\
\hline
\end{tabular}

${ }^{a}$ logarithm of the blood-brain barrier partition coefficient; ${ }^{b}$ logarithm of partition coefficient; ${ }^{\mathrm{c}}$ number of H-bond acceptors (O and $\mathrm{N}$ atoms); ${ }^{\mathrm{d}}$ number of $\mathrm{H}$-bond donors ( $\mathrm{OH}$ and $\mathrm{NH}$ groups); ${ }^{\mathrm{e}}$ topological polar surface area.

Triterpenoids exhibit antiproliferative activity against various cancer cells in vivo, though their precise molecular target it is often difficult to determine. Molecular docking studies showed significant interaction of this class compounds with important anticancer targets such as topoisomerases I and II $\alpha$, PPAR $\gamma$, EGFR, DHFR, VEGFR, NF- $\kappa \beta$, HER-2/neu, hCA-IX, CDK6 and LOX [28-33].

Molecular docking study of compound 9 was performed based on predictions of biological activity obtained with use of PASS computer program (Prediction of Activity Spectra for Substances) [34].

The predicted biological activity is determined based on the probable activity $(\mathrm{Pa})$ and probable inactivity ( $\mathrm{Pi}$ ). The values of $\mathrm{Pa}>0.7$ indicate a high probability of a specific activity, which gives a good chance to confirm it in biological studies. The PASS prediction showed anticancer activity of triterpene 9 against lung $(\mathrm{Pa}>0.81)$, melanoma $(\mathrm{Pa}>0.80)$ and breast $(\mathrm{Pa}>0.74)$ cancers in which there is an increased activity of the Akt protein. Additionally, the results obtained for compound 9 from the PASS program confirmed the studies of antiproliferative activity against Hs294T and MCF-7 cells.

The serine/threonine kinase Akt is the major signal transducer in the phosphatidylinositol 3-kinase (PI 3-K) pathway. Akt plays an important role in the cellular processes related with cancer like cell growth, proliferation, metabolism and angiogenesis. Increased expression of the Akt protein was observed in various human cancers, for example, breast, prostate, lung, ovary and brain cancer [35-37].

Molecular docking techniques were applied to investigate the binding mechanism for the series of triterpene derivatives with Akt. In our studies we retrieved the crystal structure of Akt1 from the RCSB Protein Data Bank (PDB ID: 3QKK). Docking results obtained from the GOLD program for Akt1 showed a higher degree of fit defined in the arbitrary units of the program for triterpene 9 (docking score of 57.55) compared to reference betulin 1 (docking score of 55.44). However, the alkynyl derivative 9 took a different place in the binding pocket than parent betulin 1 . The pentacyclic moiety of betulin 1 was located deep in hydrophobic cavity of CAT domain of Akt formed by Phe295/Phe299/Arg331. Significant difference in location compound 9 in CAT domain was observed.

The chemistry of the active site cavity in enzyme shows that bounded 2-butynoyl group have deeply penetrated the strong hydrophobic matrix of these proteins. Figure 3 presents the geometry of the Akt1 binding cavities, with betulin 1 and compound 9 buried in the protein's hydrophobic environment. 

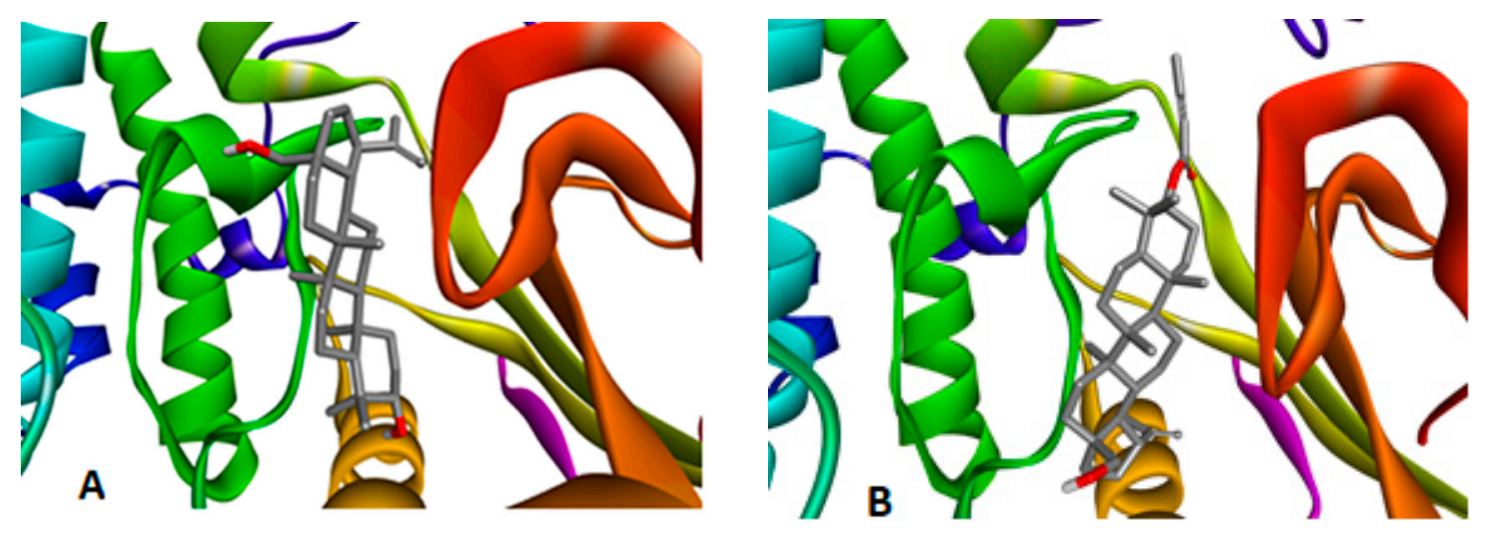

Figure 3. Akt1 stabilizing by betulin $\mathbf{1}$ (A) and compound $\mathbf{9}$ (B) docked structure. For clarity, non-polar hydrogens are invisible.

Molecular shape and hydrophobic behaviour of triterpene 9 seems to be a major factor responsible for its potent inhibitory activity (Figure 4).

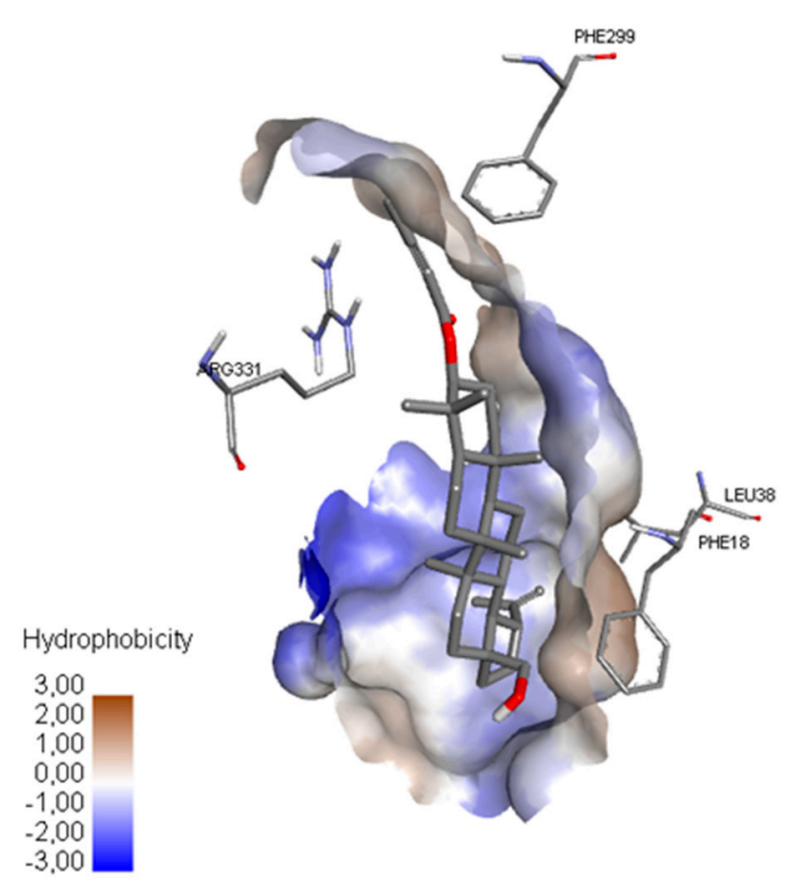

Figure 4. Hydrophobic view of the compound 9 location. For clarity, non-polar hydrogens are invisible.

Favourable hydrophobic interactions were detected between Phe18 and methyl group of the triterpene skeleton at C-8 position at the distance $4.83 \AA$ (Figure 5).

On the opposite side, Phe299 was favourably supported by interaction with the C-4 of the 2-butynoyl group at a distance $4.44 \AA$. Leu38 also exhibited hydrophobic interaction with ethenyl carbon at C-29 position, as well as Arg331 with methyl group at C-4 position at a distance 4.54 and $5.16 \AA$, respectively. 

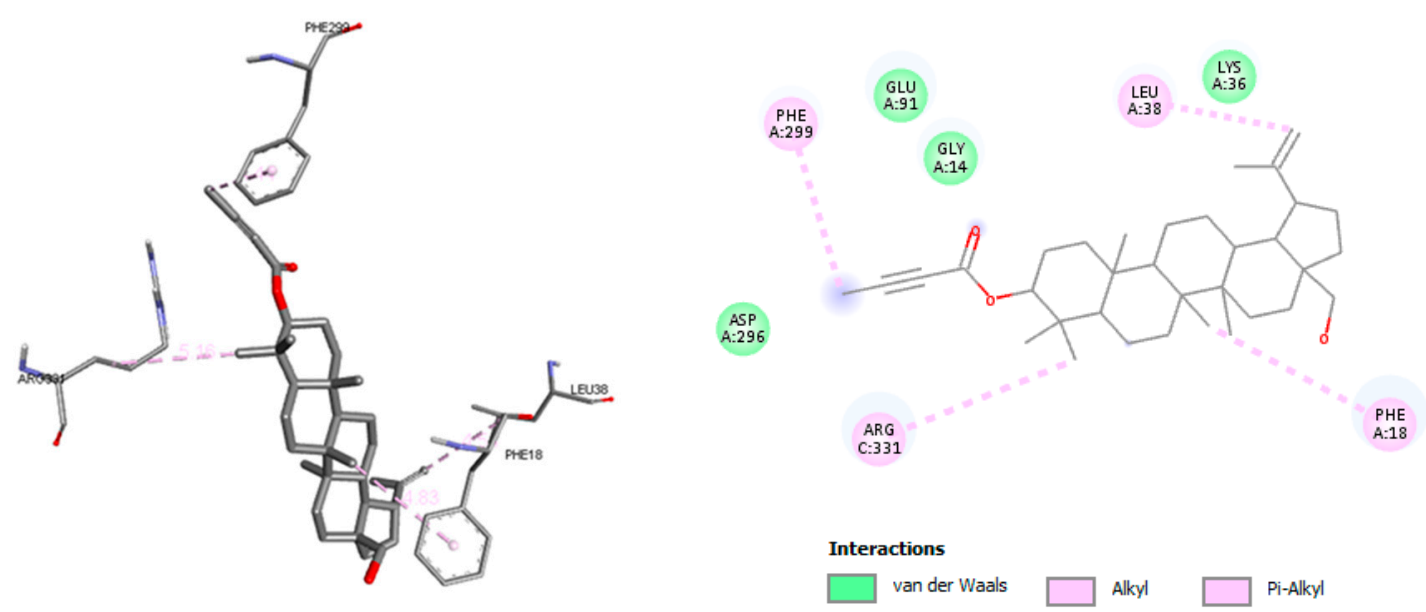

Figure 5. Binding mode of compound 9. For clarity, non-polar hydrogens are invisible.

\section{Materials and Methods}

\subsection{General Techniques}

Organic solvents were procured commercially and used after purification. Melting points (m.p.) of the obtained triterpenes were determined in open capillary tubes on an Electrothermal IA 9300 melting point apparatus and are uncorrected. Optical rotations were measured with an Atago Sac-I polarimeter (Atago, Tokyo, Japan). The NMR $\left({ }^{1} \mathrm{H}\right.$ and $\left.{ }^{13} \mathrm{C}\right)$ spectra were recorded on a Bruker Avance (Bruker, Billerica, MA, USA) III 600 spectrometer in deuterated- chloroform ( $\delta$ given in ppm, $J$ in hertz). The HR-MS of all compounds were recorded on a Bruker Impact II instrument (Bruker, Billerica, MA, USA). The IR spectra were recorded on a Shimadzu IRAffinity-1 FTIR spectrophotometer (Shimadzu, Kyoto, Japan). The progress of the reactions was monitored by thin layer chromatography (TLC) using silica gel 60 254F plates (Merck, Darmstadt, Germany). The spots were detected by spraying with a solution of $5 \%$ sulfuric (VI) acid and heating to $120{ }^{\circ} \mathrm{C}$. Silica gel $60,<63 \mu \mathrm{m}$ (Merck) were used for column chromatography. A mixture of $\mathrm{CH}_{2} \mathrm{Cl}_{2}-\mathrm{EtOH}(40: 1$ and 60:1, v/v) was applied as the mobile phase.

\subsection{Synthesis of 3-Acetylbetulin 2 and 3-Acetylbetulinic Aldehyde $\mathbf{3}$}

Synthesis of 3-acetylbetulin 2 and 3-acetylbetulinic aldehyde 3 were carried out based on the procedures described in the literature [22].

\subsection{Synthesis of 28-Tetrahydropyranylbetulin 4}

To a mixture of betulin $\mathbf{1}(0.44 \mathrm{~g}, 1 \mathrm{mmol})$ in dichloromethane $(15 \mathrm{~mL})$ was added DHP $(0.10 \mathrm{~mL}$, $1.12 \mathrm{mmol})$ and PPTS $(0.03 \mathrm{~g}, 0.12 \mathrm{mmol})$. The mixture was stirred at room temperature for 3 day in an inert gas atmosphere. Next, the resulting mixture was quenched by addition of saturated sodium bicarbonate $(5 \mathrm{~mL})$. The organic layer was washed with brine $(5 \mathrm{~mL})$, dried with anhydrous sodium sulphate and concentrated under reduced pressure. The diastereomeric mixture of ether 4 was purified by column chromatography $\left(\mathrm{CH}_{2} \mathrm{Cl}_{2}-\mathrm{EtOH} 40: 1, v / v\right)$ to give compound 4 with $57 \%$ yield $[23,24]$.

\subsection{General Procedure for the Synthesis of Betulin Derivatives 5-9}

To a mixture of ether $4(0.26 \mathrm{~g}, 0.5 \mathrm{mmol})$ and $0.55 \mathrm{mmol}$ of corresponding carboxylic acid in dichloromethane $(2.5 \mathrm{~mL})$ was added a solution of DDC $(0.11 \mathrm{~g}, 0.56 \mathrm{mmol})$ and DMAP $(0.005 \mathrm{~g}$, $0.04 \mathrm{mmol})$ in dichloromethane $(0.5 \mathrm{~mL})$ under argon atmosphere at $-10^{\circ} \mathrm{C}$ temperature. The reaction was continued to stir at $-10{ }^{\circ} \mathrm{C}$ temperature for $5 \mathrm{~h}$ and then was raised to room temperature and stirred overnight. The solvent was removed under reduced pressure. The obtained residue was dissolved in ethanol $(17 \mathrm{~mL})$ and added PPTS $(0.21 \mathrm{~g}, 0.87 \mathrm{mmml})$. The reaction mixture was agitated 
at room temperature for one week. Next, the resulting mixture was quenched by addition of saturated bicarbonate $(5 \mathrm{~mL})$ and extracted dichloromethane $(3 \times 10 \mathrm{~mL})$. The organic layer was washed with water $(4 \times 10 \mathrm{~mL})$, dried with anhydrous sodium sulphate and concentrated under reduced pressure. The crude residue was purified by column chromatography $\left(\mathrm{CH}_{2} \mathrm{Cl}_{2}-\mathrm{EtOH} 60: 1, v / v\right)$ to afford compounds 5-9 with 56-85\% yields.

3-Propanoylbetulin (5) Yield 56\%; $\mathrm{mp} \quad 252-254{ }^{\circ} \mathrm{C} ;[\alpha]_{\mathrm{D}}^{20}+1.8$ (c $\left.1, \mathrm{CHCl}_{3}\right) ; \quad \mathrm{R}_{\mathrm{f}} 0.31$ (dichloromethane/ethanol, 60:1, v/v); IR (KBr) $v_{\max } 3415,2969,1733,1465,1266 \mathrm{~cm}^{-1} ;{ }^{1} \mathrm{H}$ NMR $\left(600 \mathrm{MHz}, \mathrm{CDCl}_{3}\right): \delta 4.71(1 \mathrm{H}, \mathrm{s}, \mathrm{H}-29), 4.61(1 \mathrm{H}, \mathrm{s}, \mathrm{H}-29), 4.50(1 \mathrm{H}, \mathrm{m}, \mathrm{H}-3), 3.81(1 \mathrm{H}, \mathrm{d}, J=10.8 \mathrm{~Hz}$, $\mathrm{H}-28), 3.35(1 \mathrm{H}, \mathrm{d}, J=10.8 \mathrm{~Hz}, \mathrm{H}-28), 2.40(1 \mathrm{H}, \mathrm{m}, \mathrm{H}-19), 2.34\left(2 \mathrm{H}, \mathrm{q}, J=7.2 \mathrm{~Hz}, \mathrm{CH}_{2} \mathrm{CH}_{3}\right), 1.69(3 \mathrm{H}, \mathrm{s}$, $\left.\mathrm{CH}_{3}\right), 1.17\left(3 \mathrm{H}, \mathrm{t}, J=7.2 \mathrm{~Hz}, \mathrm{CH}_{2} \mathrm{CH}_{3}\right), 1.06\left(3 \mathrm{H}, \mathrm{s}, \mathrm{CH}_{3}\right), 1.00\left(3 \mathrm{H}, \mathrm{s}, \mathrm{CH}_{3}\right), 0.88\left(3 \mathrm{H}, \mathrm{s}, \mathrm{CH}_{3}\right), 0.87(3 \mathrm{H}$, $\left.\mathrm{s}, \mathrm{CH}_{3}\right), 0.86\left(3 \mathrm{H}, \mathrm{s}, \mathrm{CH}_{3}\right) ;{ }^{13} \mathrm{C} \mathrm{NMR}\left(150 \mathrm{MHz}, \mathrm{CDCl}_{3}\right): \delta 173.3,149.4,108.7,79.6,59.5,54.4,49.3,47.7$, $46.8,46.8,41.7,39.9,37.4,36.9,36.3,36.1,33.1,32.9,28.7,28.1,27.1,26.9,26.0,24.2,22.7,19.8,18.0,17.2$, 15.5, 15.1, 14.9, 13.7, 8.3; HRAPCIMS $m / z: 497.4002 \mathrm{C}_{33} \mathrm{H}_{53} \mathrm{O}_{3}$ (calcd. 497.3995).

3-(2-Propenoyl)betulin (6) Yield 81\%; mp 221-223 ${ }^{\circ} \mathrm{C}$; $[\alpha]_{\mathrm{D}}{ }^{20}+3.3$ (c 1, $\mathrm{CHCl}_{3}$ ); $\mathrm{R}_{\mathrm{f}} 0.33$ (dichloromethane/ethanol, 60:1, v/v); IR (KBr) $v_{\max } 3429,2943,1720,1457,1275 \mathrm{~cm}^{-1}$; ${ }^{1} \mathrm{H}$ NMR $\left(600 \mathrm{MHz}, \mathrm{CDCl}_{3}\right): \delta 6.39\left(1 \mathrm{H}, \mathrm{m}, \mathrm{CH}=\mathrm{CH}_{2}\right), 6.13\left(1 \mathrm{H}, \mathrm{m}, \mathrm{CH}=\mathrm{CH}_{2}\right), 5.82\left(1 \mathrm{H}, \mathrm{m}, \mathrm{CH}=\mathrm{CH}_{2}\right), 4.70(1 \mathrm{H}$, s, H-29), $4.61(1 \mathrm{H}, \mathrm{s}, \mathrm{H}-29), 4.58(1 \mathrm{H}, \mathrm{m}, \mathrm{H}-3), 3.82(1 \mathrm{H}, \mathrm{d}, J=10.8 \mathrm{~Hz}, \mathrm{H}-28), 3.35(1 \mathrm{H}, \mathrm{d}, J=10.8 \mathrm{~Hz}$, $\mathrm{H}-28), 2.42(1 \mathrm{H}, \mathrm{m}, \mathrm{H}-19), 1.69\left(3 \mathrm{H}, \mathrm{s}, \mathrm{CH}_{3}\right), 1.06\left(3 \mathrm{H}, \mathrm{s}, \mathrm{CH}_{3}\right), 1.01\left(3 \mathrm{H}, \mathrm{s}, \mathrm{CH}_{3}\right), 0.89\left(3 \mathrm{H}, \mathrm{s}, \mathrm{CH}_{3}\right), 0.88$ $\left(3 \mathrm{H}, \mathrm{s}, \mathrm{CH}_{3}\right), 0.87\left(3 \mathrm{H}, \mathrm{s}, \mathrm{CH}_{3}\right) ;{ }^{13} \mathrm{C}$ NMR $\left(150 \mathrm{MHz}, \mathrm{CDCl}_{3}\right): \delta 166.1,150.5,130.0,129.2,109.7,81.1$, 60.6, 55.4, 50.3, 48.8, 47.8, 47.7, 42.7, 40.9, 38.4, 38.0, 37.3, 37.1, 34.2, 34.0, 29.7, 29.2, 28.0, 27.0, 25.2, 23.7, 20.9, 20.0, 19.1, 18.2, 16.6, 16.2, 16.0, 14.7; HRAPCIMS m/z: 495.3836 $\mathrm{C}_{33} \mathrm{H}_{51} \mathrm{O}_{3}$ (calcd. 495.3838).

3-(3-Cyclopropyl-2-propynoyl)betulin (7) Yield 63\%; mp 267-269 ${ }^{\circ} \mathrm{C} ;[\alpha]_{\mathrm{D}}{ }^{20}+2.5$ (c 1, $\left.\mathrm{CHCl}_{3}\right) ; \mathrm{R}_{\mathrm{f}} 0.33$ (dichloromethane/ethanol, 60:1, v/v); IR (KBr) $v_{\max } 3530,2943,2224,1691,1452,1276 \mathrm{~cm}^{-1} ;{ }^{1} \mathrm{H}$ NMR (600 MHz, CDCl $): \delta 4.70(1 \mathrm{H}, \mathrm{s}, \mathrm{H}-29), 4.61(1 \mathrm{H}, \mathrm{s}, \mathrm{H}-29), 4.58(1 \mathrm{H}, \mathrm{m}, \mathrm{H}-3), 3.81(1 \mathrm{H}, \mathrm{d}, J=10.8 \mathrm{~Hz}$, $\mathrm{H}-28), 3.35(1 \mathrm{H}, \mathrm{d}, J=10.8 \mathrm{~Hz}, \mathrm{H}-28), 2.42(1 \mathrm{H}, \mathrm{m}, \mathrm{H}-19), 1.68\left(3 \mathrm{H}, \mathrm{s}, \mathrm{CH}_{3}\right), 1.04\left(3 \mathrm{H}, \mathrm{s}, \mathrm{CH}_{3}\right), 0.93(3 \mathrm{H}$, $\left.\mathrm{s}, \mathrm{CH}_{3}\right), 0.94-0.99\left(5 \mathrm{H}, \mathrm{m}, \mathrm{CH}, \mathrm{CH}_{2}\right), 0.89\left(3 \mathrm{H}, \mathrm{s}, \mathrm{CH}_{3}\right), 0.88\left(3 \mathrm{H}, \mathrm{s}, \mathrm{CH}_{3}\right), 0.87\left(3 \mathrm{H}, \mathrm{s}, \mathrm{CH}_{3}\right) ;{ }^{13} \mathrm{C} \mathrm{NMR}$ (150 MHz, $\mathrm{CDCl}_{3}$ ): $\delta 154.5,151.0,110.3,93.2,83.2,69.4,61.1,55.9,50.8,49.3,48.4,48.3,43.2,41.5,38.9$, 38.5, 37.8, 37.6, 34.7, 34.5, 30.3, 29.7, 28.4, 27.6, 25.7, 24.1, 21.4, 19.6, 18.7, 17.1, 16.7, 16.5, 15.2, 9.7, 1.6, HRAPCIMS m/ $z: 533.3985 \mathrm{C}_{36} \mathrm{H}_{53} \mathrm{O}_{3}$ (calcd. 533.3995).

3-Phenylpropynoylbetulin (8) Yield 83\%; mp 217-219 ${ }^{\circ} \mathrm{C}$; $[\alpha]_{\mathrm{D}}{ }^{20}+4.4$ (c $1, \mathrm{CHCl}_{3}$ ); $\mathrm{R}_{\mathrm{f}} 0.32$ (dichloromethane/ethanol, 60:1, v/v); IR (KBr) $v_{\max } 3482,2940,2218,1699,1452,1286 \mathrm{~cm}^{-1} ;{ }^{1} \mathrm{H}$ NMR $\left(600 \mathrm{MHz}, \mathrm{CDCl}_{3}\right)$ : $\delta 7.51-7.29(5 \mathrm{H}, \mathrm{m}, \mathrm{Ar}-\mathrm{H}), 4.62(1 \mathrm{H}, \mathrm{s}, \mathrm{H}-29), 4.58(1 \mathrm{H}, \mathrm{m}, \mathrm{H}-3), 4.52(1 \mathrm{H}, \mathrm{s}$, H-29), $3.72(1 \mathrm{H}, \mathrm{d}, J=10.8 \mathrm{~Hz}, \mathrm{H}-28), 3.26(1 \mathrm{H}, \mathrm{d}, J=10.8 \mathrm{~Hz}, \mathrm{H}-28), 2.32(1 \mathrm{H}, \mathrm{m}, \mathrm{H}-19), 1.67$ (3H, s, $\left.\mathrm{CH}_{3}\right), 0.98\left(3 \mathrm{H}, \mathrm{s}, \mathrm{CH}_{3}\right), 0.96\left(3 \mathrm{H}, \mathrm{s}, \mathrm{CH}_{3}\right), 0.90\left(3 \mathrm{H}, \mathrm{s}, \mathrm{CH}_{3}\right), 0.89\left(3 \mathrm{H}, \mathrm{s}, \mathrm{CH}_{3}\right), 0.84\left(3 \mathrm{H}, \mathrm{s}, \mathrm{CH}_{3}\right) ;{ }^{13} \mathrm{C}$ NMR (150 MHz, $\left.\mathrm{CDCl}_{3}\right): \delta 154.2,150.5,132.9,130.5,128.5,119.9,109.8,85.8,83.2,81.1,60.5,55.4,50.3$, 48.7, 47.8, 47.8, 42.7, 40.9, 38.4, 38.0, 37.3, 37.1, 34.1, 33.9, 29.7, 29.1, 27.9, 27.0, 25.1, 23.6, 20.9, 19.1, 18.2, 16.6, 16.2, 15.9, 14.7; HRAPCIMS $m / z: 585.3925 \mathrm{C}_{39} \mathrm{H}_{53} \mathrm{O}_{3}$ (calcd. 585.3995).

3-(2-Butynoyl)betulin (9) Yield 85\%; mp 233-236 ${ }^{\circ} \mathrm{C}$; $[\alpha]_{\mathrm{D}}{ }^{20}+1.6$ (c $\left.1, \mathrm{CHCl}_{3}\right) ; \mathrm{R}_{\mathrm{f}} 0.34$ (dichloromethane/ethanol, 60:1, v/v); IR (KBr) $\gamma_{\max } 3580,2946,2243,1699,1457,1260 \mathrm{~cm}^{-1} ;{ }^{1} \mathrm{H}$ NMR $\left(600 \mathrm{MHz}, \mathrm{CDCl}_{3}\right): \delta 4.70(1 \mathrm{H}, \mathrm{s}, \mathrm{H}-29), 4.60(1 \mathrm{H}, \mathrm{s}, \mathrm{H}-29), 4.58(1 \mathrm{H}, \mathrm{m}, \mathrm{H}-3), 3.81(1 \mathrm{H}, \mathrm{d}$, $J=10.8 \mathrm{~Hz}, \mathrm{H}-28), 3.34(1 \mathrm{H}, \mathrm{d}, J=10.8 \mathrm{~Hz}, \mathrm{H}-28), 2.44(1 \mathrm{H}, \mathrm{m}, \mathrm{H}-19), 2.00\left(3 \mathrm{H}, \mathrm{s}, \mathrm{C} \equiv \mathrm{CCH}_{3}\right), 1.70(3 \mathrm{H}, \mathrm{s}$, $\left.\mathrm{CH}_{3}\right), 1.03\left(3 \mathrm{H}, \mathrm{s}, \mathrm{CH}_{3}\right), 0.98\left(3 \mathrm{H}, \mathrm{s}, \mathrm{CH}_{3}\right), 0.89\left(3 \mathrm{H}, \mathrm{s}, \mathrm{CH}_{3}\right), 0.87\left(3 \mathrm{H}, \mathrm{s}, \mathrm{CH}_{3}\right), 0.86\left(3 \mathrm{H}, \mathrm{s}, \mathrm{CH}_{3}\right) ;{ }^{13} \mathrm{C}$ NMR $\left(150 \mathrm{MHz}, \mathrm{CDCl}_{3}\right): \delta 153.9,150.5,109.8,85.0,82.8,72.8,60.6,55.4,50.3,48.7,47.8,42.7,40.9,38.4$, 37.9, 37.3, 37.0, 34.1, 34.0, 29.7, 29.1, 27.8, 27.0, 25.1, 23.6, 20.8, 19.1, 18.2, 16.5, 16.1, 16.0, 14.7, 3.9, 1.0; HRAPCIMS $m / z: 507.3835 \mathrm{C}_{34} \mathrm{H}_{51} \mathrm{O}_{3}$ (calcd. 507.3838). 


\subsection{General Procedure for the Synthesis of Betulin Derivatives 10-14}

To a stirred mixture of ether $4(0.26 \mathrm{~g}, 0.05 \mathrm{mmol})$ in benzene $(3 \mathrm{~mL})$ in the presence of pyridine $(1.25 \mathrm{~mL})$ at $0-5{ }^{\circ} \mathrm{C}$ temperature was added solution of corresponding chloroformate $(1.5 \mathrm{mmol})$ in benzene $(2.5 \mathrm{~mL})$. The reaction was continued to stir at $0-5{ }^{\circ} \mathrm{C}$ temperature for $4 \mathrm{~h}$. Next, the reaction was raised to room temperature and stirred overnight. The reaction mixture was diluted with $2.5 \mathrm{~mL}$ of chloroform and washed with $1 \mathrm{~N}$ sulfuric acid and water. After drying with anhydrous sodium sulphate the solution was concentrated under reduced pressure. The obtained residue was dissolved in ethanol $(17 \mathrm{~mL})$ and added PPTS $(0.21 \mathrm{~g}, 0.87 \mathrm{mmml})$. The reaction mixture was agitated at room temperature for one week. Next, the resulting mixture was quenched by addition of saturated bicarbonate $(5 \mathrm{~mL})$ and extracted dichloromethane $(3 \times 10 \mathrm{~mL})$. The organic layer was washed with water $(4 \times 10 \mathrm{~mL})$, dried with anhydrous sodium sulphate and concentrated under reduced pressure. The crude residue was purified by column chromatography $\left(\mathrm{CH}_{2} \mathrm{Cl}_{2}-\mathrm{EtOH} 60: 1, v / v\right)$ to afford compounds $\mathbf{1 0}-\mathbf{1 4}$ with $67-75 \%$ yields.

3-Ethoxycarbonylbetulin (10) Yield 67\%; mp 239-242 ${ }^{\circ} \mathrm{C} ;[\alpha]_{\mathrm{D}}{ }^{20}+3.5$ (c $1, \mathrm{CHCl}_{3}$ ); $\mathrm{R}_{\mathrm{f}} 0.29$ (dichloromethane/ethanol, 60:1, v/v); IR (KBr) $v_{\max } 3497,2943,1737,1466,1269 \mathrm{~cm}^{-1}$; ${ }^{1} \mathrm{H}$ NMR $\left(600 \mathrm{MHz}, \mathrm{CDCl}_{3}\right): \delta 4.70(1 \mathrm{H}, \mathrm{s}, \mathrm{H}-29), 4.61(1 \mathrm{H}, \mathrm{s}, \mathrm{H}-29), 4.33(1 \mathrm{H}, \mathrm{m}, \mathrm{H}-3), 4.21(2 \mathrm{H}, \mathrm{q}, J=7.2 \mathrm{~Hz}$, $\left.\mathrm{OCH}_{2}\right), 3.81(1 \mathrm{H}, \mathrm{d}, J=10.8 \mathrm{~Hz}, \mathrm{H}-28), 3.35(1 \mathrm{H}, \mathrm{d}, J=10.8 \mathrm{~Hz}, \mathrm{H}-28), 2.41(1 \mathrm{H}, \mathrm{m}, \mathrm{H}-19), 1.69(3 \mathrm{H}, \mathrm{s}$, $\left.\mathrm{CH}_{3}\right), 1.31\left(3 \mathrm{H}, \mathrm{t}, J=7.2 \mathrm{~Hz}, \mathrm{CH}_{2} \mathrm{CH}_{3}\right), 1.05\left(3 \mathrm{H}, \mathrm{s}, \mathrm{CH}_{3}\right), 1.00\left(3 \mathrm{H}, \mathrm{s}, \mathrm{CH}_{3}\right), 0.94\left(3 \mathrm{H}, \mathrm{s}, \mathrm{CH}_{3}\right), 0.88(3 \mathrm{H}$, s, $\left.\mathrm{CH}_{3}\right), 0.87\left(\mathrm{~s}, \mathrm{CH}_{3}, 3 \mathrm{H}\right) ;{ }^{13} \mathrm{C} \mathrm{NMR}\left(150 \mathrm{MHz}, \mathrm{CDCl}_{3}\right): \delta 155.3,150.5,109.7,85.1,63.7,60.6,55.4,50.3$, 48.7, 47.8, 47.7, 42.7, 40.9, 38.4, 38.0, 37.3, 37.1, 34.1, 34.0, 29.7, 29.1, 27.8, 27.0, 25.2, 23.7, 20.1, 19.1, 18.1, 16.4, 16.1, 16.0, 14.7, 14.3; HRAPCIMS $m / z: 513.3940 \mathrm{C}_{33} \mathrm{H}_{53} \mathrm{O}_{4}$ (calcd. 513.3944).

3-Propoxycarbonylbetulin (11) Yield 69\%; mp 250-253 ${ }^{\circ} \mathrm{C}$; $[\alpha]_{\mathrm{D}}{ }^{20}+4.5$ (c 1, $\mathrm{CHCl}_{3}$ ); $\mathrm{R}_{\mathrm{f}} 0.37$ (dichloromethane/ethanol, 60:1, v/v); IR (KBr) $v_{\max } 3497,2953,1736,1456,1257 \mathrm{~cm}^{-1} ;{ }^{1} \mathrm{H}$ NMR $\left(600 \mathrm{MHz}, \mathrm{CDCl}_{3}\right): \delta 4.70(1 \mathrm{H}, \mathrm{s}, \mathrm{H}-29), 4.60(1 \mathrm{H}, \mathrm{s}, \mathrm{H}-29), 4.32(1 \mathrm{H}, \mathrm{m}, \mathrm{H}-3), 4.11(2 \mathrm{H}, \mathrm{t}, J=6.6 \mathrm{~Hz}$, $\left.\mathrm{OCH}_{2}\right), 3.81(1 \mathrm{H}, \mathrm{d}, J=10.8 \mathrm{~Hz}, \mathrm{H}-28), 3.35(1 \mathrm{H}, \mathrm{d}, J=10.8 \mathrm{~Hz}, \mathrm{H}-28), 2.41(1 \mathrm{H}, \mathrm{m}, \mathrm{H}-19), 1.73(2 \mathrm{H}$, $\left.\mathrm{m}, \mathrm{CH}_{2} \mathrm{CH}_{3}\right), 1.67\left(3 \mathrm{H}, \mathrm{s}, \mathrm{CH}_{3}\right), 1.08\left(3 \mathrm{H}, \mathrm{s}, \mathrm{CH}_{3}\right), 1.07\left(3 \mathrm{H}, \mathrm{s}, \mathrm{CH}_{3}\right), 1.05\left(3 \mathrm{H}, \mathrm{t}, J=7.2 \mathrm{~Hz}, \mathrm{CH}_{2} \mathrm{CH}_{3}\right)$, $1.01\left(3 \mathrm{H}, \mathrm{s}, \mathrm{CH}_{3}\right), 0.97\left(3 \mathrm{H}, \mathrm{s}, \mathrm{CH}_{3}\right), 0.87\left(\mathrm{~s}, \mathrm{CH}_{3}, 3 \mathrm{H}\right) ;{ }^{13} \mathrm{C} \mathrm{NMR}\left(150 \mathrm{MHz}, \mathrm{CDCl}_{3}\right): \delta 155.5,150.5$, 109.7, 85.1, 69.3, 60.5, 55.4, 50.3, 48.7, 47.8, 48.7, 42.7, 40.9, 38.4, 38.1, 37.3, 37.1, 34.1, 33.9, 29.7, 29.2, $27.9,27.0,25.2,23.7,22.1,19.1,18.1,16.4,16.1,16.0,14.7,10.2$; HRAPCIMS $m / z: 527.4089 \mathrm{C}_{34} \mathrm{H}_{55} \mathrm{O}_{4}$ (calcd. 527.4100).

3-Allyloxycarbonylbetulin (12) Yield 69\%; mp 220-222 ${ }^{\circ} \mathrm{C}$; $[\alpha]_{\mathrm{D}}{ }^{20}+1.7$ (c 1, $\mathrm{CHCl}_{3}$ ); $\mathrm{R}_{\mathrm{f}} 0.29$ (dichloromethane/ethanol, 60:1, v/v); IR (KBr) $v_{\max } 3501,2942,1741,1456,1253 \mathrm{~cm}^{-1} ;{ }^{1} \mathrm{H}$ NMR $\left(600 \mathrm{MHz}, \mathrm{CDCl}_{3}\right): \delta 5.97\left(1 \mathrm{H}, \mathrm{m}, \mathrm{CH}=\mathrm{CH}_{2}\right), 5.38\left(1 \mathrm{H}, \mathrm{m}, \mathrm{CH}=\mathrm{CH}_{2}\right), 5.28\left(1 \mathrm{H}, \mathrm{m}, \mathrm{CH}=\mathrm{CH}_{2}\right), 4.71(1 \mathrm{H}$, $\mathrm{s}, \mathrm{H}-29), 4.63\left(2 \mathrm{H}, \mathrm{m}, \mathrm{OCH}_{2}\right), 4.61(1 \mathrm{H}, \mathrm{s}, \mathrm{H}-29), 4.34(1 \mathrm{H}, \mathrm{m}, \mathrm{H}-3), 3.82(1 \mathrm{H}, \mathrm{d}, J=10.8 \mathrm{~Hz}, \mathrm{H}-28), 3.35$ $(1 \mathrm{H}, \mathrm{d}, J=10.8 \mathrm{~Hz}, \mathrm{H}-28), 2.41(1 \mathrm{H}, \mathrm{m}, \mathrm{H}-19), 1.67\left(3 \mathrm{H}, \mathrm{s}, \mathrm{CH}_{3}\right), 1.05\left(3 \mathrm{H}, \mathrm{s}, \mathrm{CH}_{3}\right), 1.01\left(3 \mathrm{H}, \mathrm{s}, \mathrm{CH}_{3}\right)$, $0.94\left(3 \mathrm{H}, \mathrm{s}, \mathrm{CH}_{3}\right), 0.88\left(3 \mathrm{H}, \mathrm{s}, \mathrm{CH}_{3}\right), 0.87\left(3 \mathrm{H}, \mathrm{s}, \mathrm{CH}_{3}\right) ;{ }^{13} \mathrm{C} \mathrm{NMR}\left(150 \mathrm{MHz}, \mathrm{CDCl}_{3}\right): \delta 155.1,150.5,131.9$, 118.7, 109.7, 85.5, 68.2, 60.5, 55.4, 50.3, 48.7, 47.8, 47.7, 42.7, 40.9, 38.4, 38.1, 37.3, 37.1, 34.1, 34.0, 29.7, 29.2, 27.9, 27.0, 25.2, 23.7, 20.9, 19.1, 18.1, 16.4, 16.1, 16.0, 14.7; HRAPCIMS $m / z: 525.3937 \mathrm{C}_{34} \mathrm{H}_{53} \mathrm{O}_{4}$ (calcd. 525.3944).

3-Propargyloxycarbonylbetulin (13) Yield 74\%; mp 181-182 ${ }^{\circ} \mathrm{C}$; $[\alpha]_{\mathrm{D}}{ }^{20}+1.8$ (c 1, $\mathrm{CHCl}_{3}$ ); $\mathrm{R}_{\mathrm{f}} 0.33$ (dichloromethane/ethanol, 60:1, v/v); IR (KBr) $v_{\max } 3434,3309,2945,2131,1747,1456,1254 \mathrm{~cm}^{-1}$; ${ }^{1} \mathrm{H}$ NMR $\left(600 \mathrm{MHz}, \mathrm{CDCl}_{3}\right): \delta 4.65\left(2 \mathrm{H}, \mathrm{d}, J=2.4 \mathrm{~Hz}, \mathrm{OCH}_{2}\right), 4.61(1 \mathrm{H}, \mathrm{s}, \mathrm{H}-29), 4.51(1 \mathrm{H}, \mathrm{s}, \mathrm{H}-29)$, $4.26(1 \mathrm{H}, \mathrm{m}, \mathrm{H}-3), 3.72(1 \mathrm{H}, \mathrm{d}, J=10.8 \mathrm{~Hz}, \mathrm{H}-28), 3.26(1 \mathrm{H}, \mathrm{d}, J=10.8 \mathrm{~Hz}, \mathrm{H}-28), 2.45(1 \mathrm{H}, \mathrm{t}, J=2.4 \mathrm{~Hz}$, $\mathrm{C} \equiv \mathrm{CH}), 2.31(1 \mathrm{H}, \mathrm{m}, \mathrm{H}-19), 1.67\left(3 \mathrm{H}, \mathrm{s}, \mathrm{CH}_{3}\right), 0.96\left(3 \mathrm{H}, \mathrm{s}, \mathrm{CH}_{3}\right), 0.94\left(3 \mathrm{H}, \mathrm{s}, \mathrm{CH}_{3}\right), 0.89\left(3 \mathrm{H}, \mathrm{s}, \mathrm{CH}_{3}\right)$, $0.85\left(3 \mathrm{H}, \mathrm{s}, \mathrm{CH}_{3}\right), 0.78\left(3 \mathrm{H}, \mathrm{s}, \mathrm{CH}_{3}\right) ;{ }^{13} \mathrm{C} \mathrm{NMR}\left(150 \mathrm{MHz}, \mathrm{CDCl}_{3}\right): \delta 154.6,150.5,109.8,86.3,75.4,60.5$, 55.4, 54.9, 50.3, 48.7, 47.8, 42.7, 40.9, 38.3, 38.1, 37.3, 37.0, 34.1, 33.9, 29.7, 29.1, 27.8, 27.0, 25.1, 23.6, 20.8, 19.1, 18.1, 16.4, 16.1, 16.0, 14.7; HRAPCIMS $m / z: 523.3788 \mathrm{C}_{34} \mathrm{H}_{51} \mathrm{O}_{4}$ (calcd. 523.3787). 
3-(3-Butynyloxycarbonyl)betulin (14) Yield 75\%; mp 184-186 ${ }^{\circ} \mathrm{C} ;[\alpha]_{\mathrm{D}}{ }^{20}+1.5$ (c $1, \mathrm{CHCl}_{3}$ ); $\mathrm{R}_{\mathrm{f}} 0.31$ (dichloromethane/ethanol, 60:1, v/v); IR (KBr) $v_{\max }$ 3567, 3310, 2963, 2124, 1739, 1457, $1262 \mathrm{~cm}^{-1}$; ${ }^{1} \mathrm{H}$ NMR $\left(600 \mathrm{MHz}, \mathrm{CDCl}_{3}\right): \delta 4.69(1 \mathrm{H}, \mathrm{s}, \mathrm{H}-29), 4.59(1 \mathrm{H}, \mathrm{s}, \mathrm{H}-29), 4.32(1 \mathrm{H}, \mathrm{m}, \mathrm{H}-3), 4.25(2 \mathrm{H}$, $\left.\mathrm{t}, J=7.2 \mathrm{~Hz}, \mathrm{OCH}_{2}\right), 3.81(1 \mathrm{H}, \mathrm{d}, J=10.8 \mathrm{~Hz}, \mathrm{H}-28), 3.34(1 \mathrm{H}, \mathrm{d}, J=10.8 \mathrm{~Hz}, \mathrm{H}-28), 2.60(2 \mathrm{H}, \mathrm{m}$, $\left.\mathrm{OCH}_{2} \mathrm{CH}_{2}\right), 2.40(1 \mathrm{H}, \mathrm{m}, \mathrm{H}-19), 2.02(1 \mathrm{H}, \mathrm{t}, J=2.4 \mathrm{~Hz}, \mathrm{C} \equiv \mathrm{CH}), 1.69\left(3 \mathrm{H}, \mathrm{s}, \mathrm{CH}_{3}\right), 1.08\left(3 \mathrm{H}, \mathrm{s}, \mathrm{CH}_{3}\right)$, $1.04\left(3 \mathrm{H}, \mathrm{s}, \mathrm{CH}_{3}\right), 0.99\left(3 \mathrm{H}, \mathrm{s}, \mathrm{CH}_{3}\right), 0.93\left(3 \mathrm{H}, \mathrm{s}, \mathrm{CH}_{3}\right), 0.86\left(3 \mathrm{H}, \mathrm{s}, \mathrm{CH}_{3}\right) ;{ }^{13} \mathrm{C} \mathrm{NMR}\left(150 \mathrm{MHz}, \mathrm{CDCl}_{3}\right)$ :

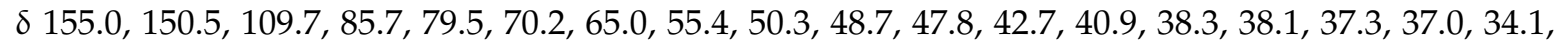
34.0, 29.7, 29.1, 27.8, 27.0, 25.1, 23.6, 20.8, 19.1, 18.1, 16.4, 16.1, 15.9, 14.7; HRAPCIMS m/z: 537.3830 $\mathrm{C}_{35} \mathrm{H}_{53} \mathrm{O}_{4}$ (calcd. 537.3944). (Supplementary data, ${ }^{1} \mathrm{H}$ NMR and ${ }^{13} \mathrm{C}$ NMR spectra of 3-modified betulin derivatives 5-14, Figures S1-S20).

\subsection{General Procedure for the Synthesis of Betulinic Aldehyde Derivatives 15-24}

To a solution of corresponding 3-substituted triterpene 5-14 $(0.22 \mathrm{mmol})$ in anhydrous dichloromethane $(3.5 \mathrm{~mL})$ was added PCC $(0.07 \mathrm{~g}, 0.33 \mathrm{mmol})$. The reaction mixture was stirred at room temperature for $2 \mathrm{~h}$. Next, the silica gel $(1 \mathrm{~g})$ was added into the mixture and stirred another $10 \mathrm{~min}$. The reaction mixture was filtered off. The filtrate was concentrated under reduced pressure and obtained residue was purified by column chromatography $\left(\mathrm{CH}_{2} \mathrm{Cl}_{2}\right.$-EtOH 60:1, v/v) to afford compounds 15-22 with $61-84 \%$ yields.

3-Propanoylbetulinic aldehyde (15) Yield 80\%; mp 155-157 ${ }^{\circ} \mathrm{C} ;[\alpha]_{\mathrm{D}}^{20}+2.3$ (c $1, \mathrm{CHCl}_{3}$ ); $\mathrm{R}_{\mathrm{f}} 0.65$ (dichloromethane/ethanol, 60:1, v/v); IR (KBr) $v_{\max } 2938,1731,1457,1260 \mathrm{~cm}^{-1} ;{ }^{1} \mathrm{H}$ NMR $(600 \mathrm{MHz}$, $\left.\mathrm{CDCl}_{3}\right): \delta 9.70(1 \mathrm{H}, \mathrm{s}, \mathrm{CHO}), 4.77(1 \mathrm{H}, \mathrm{s}, \mathrm{H}-29), 4.65(1 \mathrm{H}, \mathrm{s}, \mathrm{H}-29), 4.50(1 \mathrm{H}, \mathrm{m}, \mathrm{H}-3), 2.88(1 \mathrm{H}, \mathrm{m}, \mathrm{H}-19)$, $2.35\left(2 \mathrm{H}, \mathrm{q}, J=7.2 \mathrm{~Hz}, \mathrm{CH}_{2} \mathrm{CH}_{3}\right), 1.68\left(3 \mathrm{H}, \mathrm{s}, \mathrm{CH}_{3}\right), 1.18\left(3 \mathrm{H}, \mathrm{t}, J=7.2 \mathrm{~Hz}, \mathrm{CH}_{2} \mathrm{CH}_{3}\right), 1.00\left(3 \mathrm{H}, \mathrm{s}, \mathrm{CH}_{3}\right)$, $0.94\left(3 \mathrm{H}, \mathrm{s}, \mathrm{CH}_{3}\right), 0.87\left(3 \mathrm{H}, \mathrm{s}, \mathrm{CH}_{3}\right), 0.86\left(3 \mathrm{H}, \mathrm{s}, \mathrm{CH}_{3}\right), 0.85\left(3 \mathrm{H}, \mathrm{s}, \mathrm{CH}_{3}\right) ;{ }^{13} \mathrm{C} \mathrm{NMR}\left(150 \mathrm{MHz}, \mathrm{CDCl}_{3}\right)$ :

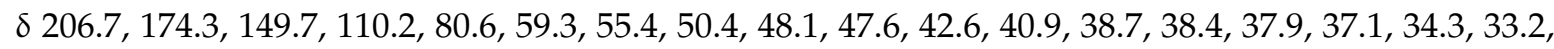
29.9, 29.2, 28.8, 28.1, 28.0, 25.5, 23.7, 20.8, 19.0, 18.2, 16.5, 16.2, 15.9, 14.3, 9.4; HRAPCIMS m/z: 495.3486 $\mathrm{C}_{33} \mathrm{H}_{51} \mathrm{O}_{3}$ (calcd. 495.3438).

3-(2-Propenoyl)betulinic aldehyde (16) Yield 83\%; mp 262-266 ${ }^{\circ} \mathrm{C} ;[\alpha]_{\mathrm{D}}{ }^{20}+3.2$ (c 1, $\mathrm{CHCl}_{3}$ ); $\mathrm{R}_{\mathrm{f}} 0.71$ (dichloromethane/ethanol, 60:1, v/v); IR (KBr) $v_{\max } 2948,1724,1452,1273 \mathrm{~cm}^{-1} ;{ }^{1} \mathrm{H} \mathrm{NMR}(600 \mathrm{MHz}$, $\left.\mathrm{CDCl}_{3}\right): \delta 9.69(1 \mathrm{H}, \mathrm{s}, \mathrm{CHO}), 6.39\left(1 \mathrm{H}, \mathrm{m}, \mathrm{CH}=\mathrm{CH}_{2}\right), 6.13\left(1 \mathrm{H}, \mathrm{m}, \mathrm{CH}=\mathrm{CH}_{2}\right), 5.82\left(1 \mathrm{H}, \mathrm{m}, \mathrm{CH}=\mathrm{CH}_{2}\right)$, $4.78(1 \mathrm{H}, \mathrm{s}, \mathrm{H}-29), 4.65(1 \mathrm{H}, \mathrm{s}, \mathrm{H}-29), 4.57(1 \mathrm{H}, \mathrm{m}, \mathrm{H}-3), 2.89(1 \mathrm{H}, \mathrm{m}, \mathrm{H}-19), 1.69\left(3 \mathrm{H}, \mathrm{s}, \mathrm{CH}_{3}\right), 1.00(3 \mathrm{H}, \mathrm{s}$, $\left.\mathrm{CH}_{3}\right), 0.90\left(3 \mathrm{H}, \mathrm{s}, \mathrm{CH}_{3}\right), 0.88\left(3 \mathrm{H}, \mathrm{s}, \mathrm{CH}_{3}\right), 0.86\left(3 \mathrm{H}, \mathrm{s}, \mathrm{CH}_{3}\right), 0.85\left(3 \mathrm{H}, \mathrm{s}, \mathrm{CH}_{3}\right) ;{ }^{13} \mathrm{C} \mathrm{NMR}(150 \mathrm{MHz}$, $\left.\mathrm{CDCl}_{3}\right): \delta 206.7,166.1,149.7,130.1,129.2,110.2,81.1,59.3,55.4,50.4,48.0,47.6,42.6,40.9,38.7,38.4$, $38.0,37.1,34.3,33.2,29.9,29.2,28.8,28.0,25.5,23.7,20.8,19.0,18.2,16.6,16.2,15.9,14.3$; HRAPCIMS m/z: $493.3326 \mathrm{C}_{33} \mathrm{H}_{49} \mathrm{O}_{3}$ (calcd. 493.3681).

3-(3-Cyclopropyl-2-propynoyl)betulinic aldehyde (17) Yield 72\%; mp 220-223 ${ }^{\circ} \mathrm{C}$; $[\alpha]_{\mathrm{D}}{ }^{20}+1.5$ (c 1 , $\mathrm{CHCl}_{3}$ ); $\mathrm{R}_{\mathrm{f}} 0.76$ (dichloromethane/ethanol, 60:1, v/v); $\mathrm{IR}(\mathrm{KBr}) v_{\max } 2939,2224,1729,1450,1250 \mathrm{~cm}^{-1}$; ${ }^{1} \mathrm{H}$ NMR $\left(600 \mathrm{MHz}, \mathrm{CDCl}_{3}\right): \delta 9.69(1 \mathrm{H}, \mathrm{s}, \mathrm{CHO}), 4.78(1 \mathrm{H}, \mathrm{s}, \mathrm{H}-29), 4.65(1 \mathrm{H}, \mathrm{s}, \mathrm{H}-29), 4.58(1 \mathrm{H}, \mathrm{m}$, $\mathrm{H}-3), 2.88(1 \mathrm{H}, \mathrm{m}, \mathrm{H}-19), 1.68\left(3 \mathrm{H}, \mathrm{s}, \mathrm{CH}_{3}\right), 1.40-1.36\left(5 \mathrm{H}, \mathrm{m}, \mathrm{CH}, \mathrm{CH}_{2}\right), 0.95\left(3 \mathrm{H}, \mathrm{s}, \mathrm{CH}_{3}\right), 0.93(3 \mathrm{H}$, $\left.\mathrm{s}, \mathrm{CH}_{3}\right), 0.89\left(3 \mathrm{H}, \mathrm{s}, \mathrm{CH}_{3}\right) 0.88\left(3 \mathrm{H}, \mathrm{s}, \mathrm{CH}_{3}\right), 0.86\left(3 \mathrm{H}, \mathrm{s}, \mathrm{CH}_{3}\right) ;{ }^{13} \mathrm{C} \mathrm{NMR}\left(150 \mathrm{MHz}, \mathrm{CDCl}_{3}\right): \delta 207.2$, 154.5, 150.2, 110.7, 93.2, 83.2, 69.4, 59.9, 56.0, 50.9, 48.6, 48.1, 43.1, 41.4, 39.2, 39.0, 38.5, 37.6, 34.8, 33.8, 30.4, 29.8, 29.3, 28.4, 26.0, 24.1, 21.3, 19.5, 18.7, 17.1, 16.7, 16.4, 14.8, 9.7, 1.6; HRAPCIMS m/z: 531.3479 $\mathrm{C}_{36} \mathrm{H}_{51} \mathrm{O}_{3}$ (calcd. 531.3838).

3-Phenylpropynoylbetulinic aldehyde (18) Yield 84\%; mp 197-199 ${ }^{\circ} \mathrm{C} ;[\alpha]_{\mathrm{D}}^{20}+2.2\left(\mathrm{c} 1, \mathrm{CHCl}_{3}\right) ; \mathrm{R}_{\mathrm{f}}$ 0.73 (dichloromethane/ethanol, 60:1, v/v); IR (KBr) $v_{\max } 2944,2228,1706,1442,1284 \mathrm{~cm}^{-1} ;{ }^{1} \mathrm{H}$ NMR (600 MHz, CDCl $): \delta 9.67(1 \mathrm{H}, \mathrm{s}, \mathrm{CHO}), 7.53-7.29(5 \mathrm{H}, \mathrm{m}, \mathrm{Ar}-\mathrm{H}), 4.69(1 \mathrm{H}, \mathrm{s}, \mathrm{H}-29), 4.58(1 \mathrm{H}, \mathrm{m}, \mathrm{H}-3)$, $4.56(1 \mathrm{H}, \mathrm{s}, \mathrm{H}-29), 2.81(1 \mathrm{H}, \mathrm{m}, \mathrm{H}-19), 1.67\left(3 \mathrm{H}, \mathrm{s}, \mathrm{CH}_{3}\right), 0.91\left(3 \mathrm{H}, \mathrm{s}, \mathrm{CH}_{3}\right), 0.88\left(3 \mathrm{H}, \mathrm{s}, \mathrm{CH}_{3}\right), 0.86(3 \mathrm{H}$, $\left.\mathrm{s}, \mathrm{CH}_{3}\right), 0.85\left(3 \mathrm{H}, \mathrm{s}, \mathrm{CH}_{3}\right), 0.79\left(3 \mathrm{H}, \mathrm{s}, \mathrm{CH}_{3}\right) ;{ }^{13} \mathrm{C} \mathrm{NMR}\left(150 \mathrm{MHz}, \mathrm{CDCl}_{3}\right): \delta 206.7,154.2,149.7,132.9$, $130.5,128.5,119.9,110.2,85.8,83.2$, 81.1, 59.4, 55.4, 53.4, 50.4, 48.0, 47.6, 42.6, 40.8, 38.7, 38.7, 38.5, 38.0, 
$37.1,34.2,33.2,29.8,29.2,28.8,27.9,25.5,23.6,20.9,20.8,19.0,18.2,16.6,16.2,15.9,14.2$; HRAPCIMS $m / z: 567.3474 \mathrm{C}_{39} \mathrm{H}_{51} \mathrm{O}_{3}$ (calcd. 567.3838).

3-(2-Butynoyl)betulinic aldehyde (19) Yield 63\%; mp 178-180 ${ }^{\circ} \mathrm{C} ;[\alpha]_{\mathrm{D}}{ }^{20}+0.8$ (c $\left.1, \mathrm{CHCl}_{3}\right) ; \mathrm{R}_{\mathrm{f}} 0.69$ (dichloromethane/ethanol, 60:1, v/v); IR (KBr) $v_{\max } 2940,2244,1697,1457,1268 \mathrm{~cm}^{-1} ;{ }^{1} \mathrm{H}$ NMR (600 MHz, CDCl 3 ): $\delta 9.60(1 \mathrm{H}, \mathrm{s}, \mathrm{CHO}), 4.69$ (1H, s, H-29), 4.56 (1H, s, H-29), 4.50 (1H, m, H-3), 2.80 $(1 \mathrm{H}, \mathrm{m}, \mathrm{H}-19), 2.02\left(3 \mathrm{H}, \mathrm{s}, \mathrm{C} \equiv \mathrm{CCH}_{3}\right), 1.69\left(3 \mathrm{H}, \mathrm{s}, \mathrm{CH}_{3}\right), 0.90\left(3 \mathrm{H}, \mathrm{s}, \mathrm{CH}_{3}\right), 0.84\left(3 \mathrm{H}, \mathrm{s}, \mathrm{CH}_{3}\right), 0.81(3 \mathrm{H}, \mathrm{s}$, $\left.\mathrm{CH}_{3}\right), 0.80\left(3 \mathrm{H}, \mathrm{s}, \mathrm{CH}_{3}\right), 0.77\left(3 \mathrm{H}, \mathrm{s}, \mathrm{CH}_{3}\right) ;{ }^{13} \mathrm{C} \mathrm{NMR}\left(150 \mathrm{MHz}, \mathrm{CDCl}_{3}\right): \delta 205.6,152.9,148.7,109.2,83.9$, 81.8, 71.8, 58.3, 54.4, 49.3, 47.0, 46.5, 41.5, 39.8, 37.6, 37.4, 36.9, 36.0, 33.2, 32.2, 28.8, 28.7, 28.2, 26.9, 24.5, 22.6, 19.7, 18.0, 17.1, 15.5, 15.2, 14.9, 13.2, 2.8; HRAPCIMS $m / z$ : 505.3684 $\mathrm{C}_{34} \mathrm{H}_{49} \mathrm{O}_{3}$ (calcd. 505.3681).

3-Ethoxycarbonylbetulinic aldehyde (20) Yield 70\%; mp 155-158 ${ }^{\circ} \mathrm{C} ;[\alpha]_{\mathrm{D}}{ }^{20}+4.1$ (c $\left.1, \mathrm{CHCl}_{3}\right) ; \mathrm{R}_{\mathrm{f}} 0.63$ (dichloromethane/ethanol, 60:1, v/v); IR (KBr) $v_{\max } 2942,1736,1465,1253 \mathrm{~cm}^{-1}$; ${ }^{1} \mathrm{H}$ NMR $(600 \mathrm{MHz}$, $\left.\mathrm{CDCl}_{3}\right): \delta 9.69(1 \mathrm{H}, \mathrm{s}, \mathrm{CHO}), 4.78(1 \mathrm{H}, \mathrm{s}, \mathrm{H}-29), 4.65(1 \mathrm{H}, \mathrm{s}, \mathrm{H}-29), 4.33(1 \mathrm{H}, \mathrm{m}, \mathrm{H}-3), 4.21(2 \mathrm{H}, \mathrm{q}$, $\left.J=7.2 \mathrm{~Hz}, \mathrm{OCH}_{2}\right), 2.88(1 \mathrm{H}, \mathrm{m}, \mathrm{H}-19), 1.70\left(3 \mathrm{H}, \mathrm{s}, \mathrm{CH}_{3}\right), 1.36\left(3 \mathrm{H}, \mathrm{t}, J=7.2 \mathrm{~Hz}, \mathrm{CH}_{2} \mathrm{CH}_{3}\right), 0.99(3 \mathrm{H}, \mathrm{s}$, $\left.\mathrm{CH}_{3}\right), 0.98\left(3 \mathrm{H}, \mathrm{s}, \mathrm{CH}_{3}\right), 0.94\left(3 \mathrm{H}, \mathrm{s}, \mathrm{CH}_{3}\right), 0.87\left(3 \mathrm{H}, \mathrm{s}, \mathrm{CH}_{3}\right), 0.86\left(\mathrm{~s}, \mathrm{CH}_{3}, 3 \mathrm{H}\right) ;{ }^{13} \mathrm{C} \mathrm{NMR}(150 \mathrm{MHz}$, $\left.\mathrm{CDCl}_{3}\right): \delta 206.7,155.3,149.7,110.2,85.1,63.7,59.3,55.4,50.4,48.0,47.6,42.6,40.8,38.7,38.4,38.0,37.1$, 34.2, 33.2, 29.9, 29.2, 28.8, 27.9, 25.5, 23.7, 20.8, 19.0, 18.1, 16.4, 16.2, 15.9, 14.3, 14.2; HRAPCIMS $m / z$ : 511.3429 $\mathrm{C}_{33} \mathrm{H}_{51} \mathrm{O}_{4}$ (calcd. 511.3787).

3-Propoxycarbonylbetulinic aldehyde (21) Yield 75\%; mp 168-171 ${ }^{\circ} \mathrm{C} ;[\alpha]_{\mathrm{D}}{ }^{20}+1.1$ (c $\left.1, \mathrm{CHCl}_{3}\right) ; \mathrm{R}_{\mathrm{f}} 0.69$ (dichloromethane/ethanol, 60:1, v/v); IR (KBr) $v_{\max } 2941,1735,1465,1266 \mathrm{~cm}^{-1} ;{ }^{1} \mathrm{H} \mathrm{NMR}(600 \mathrm{MHz}$, $\left.\mathrm{CDCl}_{3}\right): \delta 9.69(1 \mathrm{H}, \mathrm{s}, \mathrm{CHO}), 4.77(1 \mathrm{H}, \mathrm{s}, \mathrm{H}-29), 4.65(1 \mathrm{H}, \mathrm{s}, \mathrm{H}-29), 4.33(1 \mathrm{H}, \mathrm{m}, \mathrm{H}-3), 4.11(2 \mathrm{H}, \mathrm{t}$, $\left.J=6.6 \mathrm{~Hz}, \mathrm{OCH}_{2}\right), 2.89(1 \mathrm{H}, \mathrm{m}, \mathrm{H}-19), 1.74\left(2 \mathrm{H}, \mathrm{m}, \mathrm{CH}_{2} \mathrm{CH}_{3}\right), 1.70\left(3 \mathrm{H}, \mathrm{s}, \mathrm{CH}_{3}\right), 0.99\left(3 \mathrm{H}, \mathrm{s}, \mathrm{CH}_{3}\right), 0.97$ $\left(3 \mathrm{H}, \mathrm{t}, J=7.2 \mathrm{~Hz}, \mathrm{CH}_{2} \mathrm{CH}_{3}\right), 0.93\left(3 \mathrm{H}, \mathrm{s}, \mathrm{CH}_{3}\right), 0.92\left(3 \mathrm{H}, \mathrm{s}, \mathrm{CH}_{3}\right), 0.87\left(\mathrm{~s}, \mathrm{CH}_{3}, 3 \mathrm{H}\right), 0.86\left(\mathrm{~s}, \mathrm{CH}_{3}, 3 \mathrm{H}\right) ;{ }^{13} \mathrm{C}$ NMR (150 MHz, $\left.\mathrm{CDCl}_{3}\right)$ : $\delta$ 205.7, 154.4, 148.7, 109.2, 84.1, 68.2, 58.3, 54.4, 49.3, 47.0, 46.5, 41.5, 39.8, 37.7, 37.0, 36.0, 33.2, 32.2, 28.8, 28.7, 28.2, 27.8, 26.8, 24.5, 22.7, 21.0, 19.7, 18.0, 15.4, 15.2, 13.2, 9.2; HRAPCIMS $m / z: 525.3601 \mathrm{C}_{34} \mathrm{H}_{53} \mathrm{O}_{4}$ (calcd. 525.3944).

3-Allyloxycarbonylbetulinic aldehyde (22) Yield 75\%; mp 142-145 ${ }^{\circ} \mathrm{C} ;[\alpha]_{\mathrm{D}}{ }^{20}+2.1\left(\mathrm{c} 1, \mathrm{CHCl}_{3}\right) ; \mathrm{R}_{\mathrm{f}} 0.73$ (dichloromethane/ethanol, 60:1, v/v); IR (KBr) $v_{\max } 2941,1739,1458,1271 \mathrm{~cm}^{-1} ;{ }^{1} \mathrm{H}$ NMR $(600 \mathrm{MHz}$, $\left.\mathrm{CDCl}_{3}\right): \delta 9.60(1 \mathrm{H}, \mathrm{s}, \mathrm{CHO}), 5.88\left(1 \mathrm{H}, \mathrm{m}, \mathrm{CH}=\mathrm{CH}_{2}\right), 5.29\left(1 \mathrm{H}, \mathrm{m}, \mathrm{CH}=\mathrm{CH}_{2}\right), 5.20\left(1 \mathrm{H}, \mathrm{m}, \mathrm{CH}=\mathrm{CH}_{2}\right)$, $4.68(1 \mathrm{H}, \mathrm{s}, \mathrm{H}-29), 4.56\left(2 \mathrm{H}, \mathrm{m}, \mathrm{OCH}_{2}\right), 4.55(1 \mathrm{H}, \mathrm{s}, \mathrm{H}-29), 4.25(1 \mathrm{H}, \mathrm{m}, \mathrm{H}-3), 2.80(1 \mathrm{H}, \mathrm{m}, \mathrm{H}-19), 1.69(3 \mathrm{H}$, $\left.\mathrm{s}, \mathrm{CH}_{3}\right), 0.91\left(3 \mathrm{H}, \mathrm{s}, \mathrm{CH}_{3}\right), 0.90\left(3 \mathrm{H}, \mathrm{s}, \mathrm{CH}_{3}\right), 0.78\left(3 \mathrm{H}, \mathrm{s}, \mathrm{CH}_{3}\right), 0.77\left(3 \mathrm{H}, \mathrm{s}, \mathrm{CH}_{3}\right), 0.71\left(3 \mathrm{H}, \mathrm{s}, \mathrm{CH}_{3}\right) ;{ }^{13} \mathrm{C}$ NMR $\left(150 \mathrm{MHz}, \mathrm{CDCl}_{3}\right): \delta 206.7,155.1,149.7,131.9,118.7,110.2,85.5,68.2,59.3,55.4,50.4,48.0,47.5$, 42.6, 40.8, 38.7, 38.4, 38.0, 37.1, 34.2, 33.2, 29.8, 29.2, 28.8, 27.9, 25.5, 23.6, 20.8, 19.0, 18.1, 16.4, 16.2, 15.9, 14.3; HRAPCIMS m/ z: $539.3763 \mathrm{C}_{34} \mathrm{H}_{51} \mathrm{O}_{4}$ (calcd. 539.3787).

3-Propargyloxycarbonylbetulinic aldehyde (23) Yield 81\%; mp $145-147{ }^{\circ} \mathrm{C} ;[\alpha]_{\mathrm{D}}{ }^{20}+2.5\left(\mathrm{c} 1, \mathrm{CHCl}_{3}\right)$; $\mathrm{R}_{\mathrm{f}} 0.72$ (dichloromethane/ethanol, 60:1, v/v); IR (KBr) $v_{\max } 3311,2941,2131,1747,1456,1274 \mathrm{~cm}^{-1}$; ${ }^{1} \mathrm{H}$ NMR $\left(600 \mathrm{MHz}, \mathrm{CDCl}_{3}\right): \delta 9.67(1 \mathrm{H}, \mathrm{s}, \mathrm{CHO}), 4.75(1 \mathrm{H}, \mathrm{s}, \mathrm{H}-29), 4.71\left(2 \mathrm{H}, \mathrm{d}, J=2.4 \mathrm{~Hz}, \mathrm{OCH}_{2}\right), 4.63$ $(1 \mathrm{H}, \mathrm{s}, \mathrm{H}-29), 4.33(1 \mathrm{H}, \mathrm{m}, \mathrm{H}-3), 2.86(1 \mathrm{H}, \mathrm{m}, \mathrm{H}-19), 2.51(1 \mathrm{H}, \mathrm{t}, J=2.4 \mathrm{~Hz}, \mathrm{C} \equiv \mathrm{CH}), 1.69\left(3 \mathrm{H}, \mathrm{s}, \mathrm{CH}_{3}\right)$, $0.97\left(3 \mathrm{H}, \mathrm{s}, \mathrm{CH}_{3}\right), 0.92\left(3 \mathrm{H}, \mathrm{s}, \mathrm{CH}_{3}\right), 0.91\left(3 \mathrm{H}, \mathrm{s}, \mathrm{CH}_{3}\right), 0.85\left(3 \mathrm{H}, \mathrm{s}, \mathrm{CH}_{3}\right), 0.84\left(3 \mathrm{H}, \mathrm{s}, \mathrm{CH}_{3}\right) ;{ }^{13} \mathrm{C} \mathrm{NMR}$ (150 MHz, $\mathrm{CDCl}_{3}$ ): $\delta$ 206.6, 154.6, 149.7, 110.2, 86.2, 77.2, 76.8, 75.4, 59.3, 55.4, 54.9, 50.3, 48.0, 47.5, 42.6, 40.8, 38.7, 38.3, 38.0, 37.0, 34.2, 33.2, 29.8, 29.2, 28.8, 27.8, 25.5, 23.6, 20.8, 19.0, 18.1, 16.3, 16.1, 15.9, 14.2; HRAPCIMS m/z: $521.3643 \mathrm{C}_{34} \mathrm{H}_{49} \mathrm{O}_{4}$ (calcd. 521.3631).

3-(3-Butynyloxycarbonyl)betulinic aldehyde (24) Yield 61\%; mp $143-145{ }^{\circ} \mathrm{C} ;[\alpha]_{\mathrm{D}}{ }^{20}+3.3\left(\mathrm{c} 1, \mathrm{CHCl}_{3}\right)$; $\mathrm{R}_{\mathrm{f}} 0.68$ (dichloromethane/ethanol, 60:1, v/v); $\mathrm{IR}(\mathrm{KBr}) v_{\max } 3284,2940,2358,1746,1456,1274 \mathrm{~cm}^{-1}$; ${ }^{1} \mathrm{H}$ NMR $\left(600 \mathrm{MHz}, \mathrm{CDCl}_{3}\right): \delta 9.69(1 \mathrm{H}, \mathrm{s}, \mathrm{CHO}), 4.77(1 \mathrm{H}, \mathrm{s}, \mathrm{H}-29), 4.65(1 \mathrm{H}, \mathrm{s}, \mathrm{H}-29), 4.33(1 \mathrm{H}, \mathrm{m}$, $\mathrm{H}-3), 4.24\left(2 \mathrm{H}, \mathrm{t}, J=7.2 \mathrm{~Hz}, \mathrm{OCH}_{2}\right), 2.88(1 \mathrm{H}, \mathrm{m}, \mathrm{H}-19), 2.60\left(2 \mathrm{H}, \mathrm{m}, \mathrm{OCH}_{2} \mathrm{CH}_{2}\right), 2.02(1 \mathrm{H}, \mathrm{t}, J=2.4 \mathrm{~Hz}$, $\mathrm{C} \equiv \mathrm{CH}), 1.71\left(3 \mathrm{H}, \mathrm{s}, \mathrm{CH}_{3}\right), 0.99\left(3 \mathrm{H}, \mathrm{s}, \mathrm{CH}_{3}\right), 0.93\left(3 \mathrm{H}, \mathrm{s}, \mathrm{CH}_{3}\right), 0.86\left(3 \mathrm{H}, \mathrm{s}, \mathrm{CH}_{3}\right), 0.80\left(3 \mathrm{H}, \mathrm{s}, \mathrm{CH}_{3}\right), 0.77$ $\left(3 \mathrm{H}, \mathrm{s}, \mathrm{CH}_{3}\right) ;{ }^{13} \mathrm{C}$ NMR $\left(150 \mathrm{MHz}, \mathrm{CDCl}_{3}\right): \delta 206.7,155.0,149.7,110.2,85.7,70.2,65.0,59.3,47.5,42.6$, $40.8,38.1,37.1,33.2,29.8,29.2,28.8,27.8,25.5,23.6,20.8,19.1,19.0,18.1,16.4,16.2,15.9,14.2 ;$ EIMS $m / z$ 
$536[\mathrm{M}]^{+}$(14), 189 (100). (Supplementary data, ${ }^{1} \mathrm{H}$ NMR and ${ }^{13} \mathrm{C}$ NMR spectra of 3-modified betulinic aldehyde derivatives 15-24, Figures S21-S40).

\subsection{Antiproliferative Activity—MTT and SRB Assays}

\subsubsection{Human Cell Lines}

The applied human cell lines such as MV-4-11 (biphenotypic B myelomonocytic leukaemia), A549 (lung adenocarcinoma), Du-145 (prostate), Hs294T (melanoma), MCF-7 (breast adenocarcinoma) and MCF-10A (normal mammary gland) were obtained from the American Type Culture Collection (ATCC, Rockville, MD, USA). The all cell lines are maintained at the Cell Culture Collection of the Institute of Immunology and Experimental Therapy (Wrocław, Poland). The cells were plated in 96-well plates (Sarstedt, Newton, MA, USA) at a density of $1 \times 10^{4}$ cells per well in $100 \mu \mathrm{L}$ of appropriate culture medium overnight.

Biphenotypic B myelomonocytic leukaemia (MV-4-11) cells were cultured in RPMI 1640 medium (Gibco, Scotland, UK) with GlutaMAX (Thermo-Fisher Scientific, Warsaw, Poland) adjusted to contain $1.0 \mathrm{mM}$ sodium pyruvate (Sigma-Aldrich, Chemie $\mathrm{GmbH}$, Steinheim, Germany). Human lung adenocarcinoma (A549) cells were cultured in mixture RPMI 1640 and Opti-MEM medium (Gibco, Scotland, UK) supplemented with GlutaMAX (ThermoFisher Scientific, Warsaw, Poland) and 2mM L-glutamine adjusted to contain $1.0 \mathrm{mM}$ sodium pyruvate. Human prostate (Du-145) and human breast adenocarcinoma (MCF-7) cells were cultured in Eagle medium (IIET, Wrocław, Poland), supplemented with $2 \mathrm{mM}$ L-glutamine adjusted to contain $1.0 \mathrm{mM}$ sodium pyruvate. Human melanoma (Hs294T) cells were cultured in Dulbecco medium (Gibco, Scotland, UK) supplemented with $2 \mathrm{mM}$ L-glutamine. The normal human mammary gland cells (MCF-10A) were cultured in Ham's F-12 medium containing $2 \mathrm{mM}$ L-glutamine, $5 \%$ horse serum, epidermal growth factor EGF $(100 \mu \mathrm{L} / 100 \mathrm{~mL}$ of medium), hydrocortisone $(1 \mathrm{~mL} / 100 \mathrm{~mL}$ of medium), insulin $(100 \mu \mathrm{L} / 100 \mathrm{~mL}$ of medium) and cholera toxin $(0.1 \mathrm{~mL} / 100 \mathrm{~mL}$ of medium). All applied culture media except MCF-10A were supplemented with $10 \%$ of FBS (MV-4-11 cultured with FBS derived from Sigma-Aldrich, Chemie GmbH, Steinheim, Germany, the other cell lines FBS HyClone Thermo-Fisher Scientific, Warsaw, Poland) and streptomycin $(100 \mu \mathrm{g} / \mathrm{mL})$, penicillin $(100 \mathrm{U} / \mathrm{mL})$ (both from Polfa, Tarchomin, Poland). The tested cell lines were grown at $37^{\circ} \mathrm{C}$ in humid atmosphere saturated with $5 \% \mathrm{CO}_{2}$.

\subsubsection{Antiproliferative Assays}

In our studies were used two assays for determination the cytotoxic activity in vitro of triterpene derivatives. By dissolving $1 \mathrm{mg}$ of each tested compound in $100 \mu \mathrm{L}$ of DMSO, stock solutions at a $10 \mathrm{mg} / \mathrm{mL}$ were obtained. Next, the stock solutions were diluted in appropriate culture medium to reach the required concentrations of compounds ranging from 0.1 to $100 \mu \mathrm{g} / \mathrm{mL}$. The MTT assay was used for the cytotoxicity screening against biphenotypic B myelomonocytic leukaemia (MV-4-11). The SRB assay was performed for adherent cells such as A549, Du-145, Hs294T, MCF-7 and MCF-10A. Both assays were carried as described by Wietrzyk et al. [38]. The triterpenes in given concentration were examined in triplicates in each experiment, which was repeated 3 times. The results of anticancer activity in vitro were presented as an $\mathrm{IC}_{50}$ in $\mu \mathrm{M}$. Cisplatin and betulin $\mathbf{1}$ were used as reference substances.

\subsection{In Silico Study}

The physicochemical parameters of compounds 5-24 such as penetration drugs by BBB (log BB), lipophilicity (cLogP), topological polar surface area (tPSA), hydrogen bond donors (HBD) and hydrogen bond acceptors (HBA) topological polar surface area (tPSA) were calculated using the ACD/Labs software [39]. 
The target macromolecule for molecular docking studies were used from the Protein Data Bank (available online: https://www.rcsb.org/). In our studies we applied 3D crystal structure of Akt1 (ID: 3QKK).

The three-dimensional (3D) structures of tested triterpenes required were generated in their low-energy conformation using Gaussian 16 (revision A.03) computer code [40] at the density functional theory (DFT, B3LYP [41]) and 6-311 + G(d,p) basis sets. In docking study was used Genetic Optimisation for Ligand Docking (GOLD) 5.6.3 [42] The receptors for docking were prepared using a Gold packet of the Hermes visualizer. The docking was defined for all the protein residues within $10 \AA$ of the reference ligands that accompanied the downloaded protein complexes. For all other parameters a default values were used and the complexes were submitted to 10 genetic algorithm runs using the GOLDScore fitness function. The BIOVIA Discovery Studio virtual environment was used to visualize molecular docking details [43].

\section{Conclusions}

The synthesis, biological activity and in silico studies of newly 3-substituted derivatives of betulin and betulinic aldehyde were presented. The molecular structures of triterpenes 5-24 were confirmed by ${ }^{1} \mathrm{H}$ NMR, ${ }^{13} \mathrm{C}$ NMR, IR and HR-MS spectra. Antiproliferative activity of compounds was evaluated in vitro against five human cancer cell lines such as biphenotypic B myelomonocytic leukaemia (MV-4-11), adenocarcinoma (A549), prostate (Du-145), melanoma (Hs294T), breast adenocarcinoma (MCF-7) and normal human mammary gland (MCF-10A). The compound 9 having 2-butynoyl group, showed the highest anticancer activity against three tested cancer cell lines: Du-145, Hs294T and MCF-7. Molecular docking studies were performed to investigate the binding mechanism of the 3-substituted derivatives of betulin and betulinic aldehyde with Akt protein. Our studies showed significant difference in location of triterpene 9 in Akt CAT domain when compared to betulin 1. Moreover, the hydrophobic behaviour of compound 9 may be explanation of its potent inhibitor activity.

Supplementary Materials: Supplementary materials can be found at http:/ /www.mdpi.com/1422-0067/20/6/ 1372/s1.

Author Contributions: E.B. and S.B. developed the concept of the work. E.B. performed experiments, analysed spectra and wrote the paper. E.C. and M.K.-T. contributed to the purification of all compounds. K.M. performed and described the molecular docking study. J.T. and J.W. conducted a study of the biological activity.

Acknowledgments: This work was supported by the Medical University of Silesia (grant KNW-1-072/K/8/O). The calculations have been performed using resources provided by Wroclaw Centre for Networ- 800 king and Supercomputing (http:/ / wcss.pl), Grant No. 382.

Conflicts of Interest: The authors declare no conflict of interest.

\section{Abbreviations}

$\begin{array}{ll}\text { Akt } & \text { serine/threonine protein kinase } \\ \text { ADME } & \text { absorption, distribution, metabolism and excretion } \\ \text { PPAR } \gamma & \text { peroxisome proliferator-activated receptor } \\ \text { EGFR } & \text { epidermal growth factor receptor } \\ \text { DHFR } & \text { dihydrofolate reductase } \\ \text { VEGFR } & \text { vascular endothelial growth factor } \\ \text { NF- } \beta & \text { transcription factor } \\ \text { HER-2/neu } & \text { epidermal growth factor receptor EGFR family } \\ \text { hCA-IX } & \text { human carbonic anhydrase IX } \\ \text { CDK6 } & \text { cyclin-dependent kinase 6 } \\ \text { LOX } & \text { lipoxygenase }\end{array}$




\section{References}

1. Naman, C.B.; Benatrehina, P.A.; Kinghorn, A.D. Pharmaceuticals, Plant Drugs. In Encyclopedia of Applied Plant Sciences, 2nd ed.; Thomas, B., Murray, B.G., Murphy, D.J., Eds.; Elsevier: Amsterdam, The Netherlands, 2017; Volume 2, pp. 93-99. [CrossRef]

2. Ngoc, T.D.; Moons, N.; Kim, Y.; De Borggraeve, W.; Mashentseva, A.; Andrei, G.; Snoeck, R.; Balzarini, J.; Dehaen, W. Synthesis of triterpenoid triazine derivatives from allobetulone and betulonic acid with biological activities. Bioorg. Med. Chem. 2014, 22, 3292-3300. [CrossRef] [PubMed]

3. Yang, S.J.; Liu, M.C.; Zhao, Q.; Hu, D.Y.; Xue, W.; Yang, S. Synthesis and biological evaluation of betulonic acid derivatives as antitumor agents. Eur. J. Med. Chem. 2015, 96, 58-65. [CrossRef] [PubMed]

4. Gupta, N.; Rath, S.K.; Singh, J.; Qayum, A.; Singh, S.; Sangwan, P.L. Synthesis of novel benzylidene analogues of betulinic acid as potent cytotoxic agents. Eur. J. Med. Chem. 2017, 135, 517-530. [CrossRef] [PubMed]

5. Li, J.; Goto, M.; Yang, X.; Susan, L.; Morris-Natschke, S.L.; Huang, L.; Chen, C.H.; Lee, K.H. Fluorinated betulinic acid derivatives and evaluation of their anti-HIV activity. Bioorg. Med. Chem. Lett. 2016, 26, 68-71. [CrossRef] [PubMed]

6. Xiao, S.; Wang, Q.; Si, L.; Zhou, X.; Zhang, Y.; Zhang, L.; Zhou, D. Synthesis and biological evaluation of novel pentacyclic triterpene $\alpha$-cyclodextrin conjugates as HCV entry inhibitors. Eur. J. Med. Chem. 2016, 124, 1-9. [CrossRef] [PubMed]

7. Haque, S.; Nawrot, D.A.; Alakurtti, S.; Ghemtio, L.; Yli-Kauhaluoma, J.; Tammela, P. Screening and characterisation of antimicrobial properties of semisynthetic betulin derivatives. PLoS ONE 2014, 9, e102696. [CrossRef]

8. Medvedeva, N.I.; Kazakova, O.B.; Lopatina, T.V.; Smirnova, I.E.; Giniyatullina, G.V.; Baikova, I.P.; Kataev, V.E. Synthesis and antimycobacterial activity of triterpenic A-ring azepanes. Eur. J. Med. Chem. 2018, 143, 464-472. [CrossRef]

9. Schwiebs, A.; Radeke, H.H. Immunopharmacological activity of betulin in inflammation-associated carcinogenesis. Anticancer Agents Med. Chem. 2018, 18, 645-651. [CrossRef]

10. Begum, R.; Sheliya, M.A.; Mir, S.R.; Singh, E.; Sharma, M. Inhibition of proinflammatory mediators by coumaroyl lupendioic acid, a new lupane-type triterpene from Careya arborea, on inflammation-induced animal model. J. Ethnopharmacol. 2017, 206, 376-392. [CrossRef]

11. Jingbo, W.; Aimin, C.; Qi, W.; Xin, L.; Huaining, L. Betulinic acid inhibits IL-1 $\beta$-induced inflammation by activating PPAR- $\gamma$ in human osteoarthritis chondrocytes. Int. Immunopharmacol. 2015, 29, 687-692. [CrossRef]

12. Kvasnica, M.; Sarek, J.; Klinotova, E.; Dzubak, P.; Hajduch, M. Synthesis of phthalates of betulinic acid and betulin with cytotoxic activity. Bioorg. Med. Chem. 2005, 13, 3447-3454. [CrossRef]

13. Ahmad, F.B.H.; Moghaddam, M.G.; Basri, M.; Rahman, M.B.A. Anticancer activity of 3-O-acylated betulinic acid derivatives obtained by enzymatic synthesis. Biosci. Biotechnol. Biochem. 2010, 74, 1025-1029. [CrossRef]

14. Rajendran, P.; Jaggi, M.; Singh, M.K.; Mukherjee, R.; Burman, A.C. Pharmacological evaluation of C-3 modified betulinic acid derivatives with potent anticancer activity. Investig. New Drugs 2008, 26, 25-34. [CrossRef]

15. Santos, R.C.; Salvador, J.A.R.; Marín, S.; Cascante, M.; Moreira, J.N.; Dinis, T.C.P. Synthesis and structure-activity relationship study of novel cytotoxic carbamate and $\mathrm{N}$-acylheterocyclic bearing derivatives of betulin and betulinic acid carbamate and $\mathrm{N}$-acylheterocyclic bearing derivatives of betulin and betulinic acid. Bioorg. Med. Chem. 2010, 18, 4385-4396. [CrossRef] [PubMed]

16. Gauthier, C.; Legault, J.; Lebrun, M.; Dufour, P.; Pichette, A. Glycosidation of lupane-type triterpenoids as potent in vitro cytotoxic agents. Bioorg. Med. Chem. 2006, 14, 6713-6725. [CrossRef] [PubMed]

17. Cui, H.W.; He, Y.; Wang, J.; Gao, W.; Liu, T.; Qin, M.; Wang, X.; Gao, C.; Wang, Y.; Liu, M.Y.; et al. Synthesis of heterocycle-modified betulinic acid derivatives as antitumor agents. Eur. J. Med. Chem. 2015, 95, 240-248. [CrossRef] [PubMed]

18. Bębenek, E.; Jastrzębska, M.; Kadela-Tomanek, M.; Chrobak, E.; Orzechowska, B.; Zwolińska, K.; Latocha, M.; Mertas, A.; Czuba, Z.; Boryczka, S. Novel triazole hybrids of betulin: Synthesis and biological activity profile. Molecules 2017, 22, 1876. [CrossRef] 
19. Bębenek, E.; Kadela-Tomanek, M.; Chrobak, E.; Wietrzyk, J.; Sadowska, J.; Boryczka, S. New acetylenic derivatives of betulin and betulone, synthesis and cytotoxic activity. Med. Chem. Res. 2017, 26, 1-8. [CrossRef] [PubMed]

20. Bębenek, E.; Kadela-Tomanek, M.; Chrobak, E.; Latocha, M.; Boryczka, S. Novel triazoles of 3-acetylbetulin and betulone as anticancer agents. Med. Chem. Res. 2018, 27, 2051-2061. [CrossRef] [PubMed]

21. Chrobak, E.; Bębenek, E.; Kadela-Tomanek, M.; Latocha, M.; Jelsch, C.; Wenger, E.; Boryczka, S. Betulin phosphonates; synthesis, structure, and cytotoxic activity. Molecules 2016, 21, 1123. [CrossRef]

22. Liu, S.; Xu, F.; Liu, L.; Ma, D. A convenient synthesis of lupeol from betulin. Ind. Crop. Prod. 2015, 74, 494-496. [CrossRef]

23. Kim, D.S.H.L.; Chen, Z.; Nguyen, T.; Pezzuto, J.M.; Qiu, S.; Lu, Z.Z. A concise semi-synthetic approach to betulinic acid from betulin. Synthetic. Commun. 1997, 27, 1607-1612. [CrossRef]

24. Pohjala, L.; Alakurtti, S.; Ahola, T.; Yli-Kauhaluoma, J.; Tammela, P. Betulin-derived compounds as inhibitors of alphavirus replication. J. Nat. Prod. 2009, 72, 1917-1926. [CrossRef] [PubMed]

25. Rostom, S.A.F.; Badr, M.H.; Abd El Razik, H.A.; Ashour, H.M.A. Structure-based development of novel triazoles and related thiazolotriazoles as anticancer agents and Cdc25A/B phosphatase inhibitors. Synthesis, in vitro biological evaluation, molecular docking and in silico ADME-T studies. Eur. J. Med. Chem. 2017, 139, 263-279. [CrossRef] [PubMed]

26. Trifunović, J.; Borčić, V.; Vukmirović, S.; Goločorbin Kon, S.; Mikov, M. Retention data of bile acids and their oxo derivatives in characterization of pharmacokinetic properties and in silico ADME modeling. Eur. J. Pharm. Sci. 2016, 92, 194-202. [CrossRef]

27. Tzvetkov, N.T.; Stammler, H.G.; Neumann, B.; Hristova, S.; Antonov, L.; Gastreich, M. Crystal structures, binding interactions, and ADME evaluation of brain penetrant $\mathrm{N}$-substituted indazole-5-carboxamides as subnanomolar, selective monoamine oxidase B and dual MAO-A/B inhibitors. Eur. J. Med. Chem. 2017, 127, 470-492. [CrossRef] [PubMed]

28. Saeed, A.; Hussain, H.; Shamraiz, U.; Rehman, N.U.; Khan, H.Y.; Badshah, A.; Heller, L.; Csuk, R.; Ali, M.; Khan, A.; et al. Synthesis of new triterpenic monomers and dimers as potential antiproliferative agents and their molecular docking studies. Eur. J. Med. Chem. 2018, 143, 948-957. [CrossRef]

29. Sathya, S.; Sudhagar, S.; Sarathkumar, B.; Lakshmi, B.S. EGFR inhibition by pentacyclic triterpenes exhibit cell cycle and growth arrest in breast cancer cells. Life Sci. 2014, 95, 53-62. [CrossRef]

30. Bhandari, P.; Patel, N.K.; Gangwal, R.P.; Sangamwar, A.T.; Bhutani, K.K. Oleanolic acid analogs as NO, TNF- $\alpha$ and IL-1 $\beta$ inhibitors: Synthesis, biological evaluation and docking studies. Bioorg. Med. Chem. Lett. 2014, 24, 4114-4119. [CrossRef]

31. Ashour, A.; El-Sharkawy, S.; Amer, M.; Bar, F.A.; Katakura, Y.; Miyamoto, T.; Toyota, N.; Bang, T.H.; Kondo, R.; Shimizu, K. Rational design and synthesis of topoisomerase I and II inhibitors based on oleanolic acid moiety for new anti-cancer drugs. Bioorg. Med. Chem. 2014, 22, 211-220. [CrossRef]

32. Wang, L.; Waltenberger, B.; Pferschy-Wenzig, E.M.; Blunder, M.; Liu, X.; Malainer, C.; Blazevic, T.; Schwaiger, S.; Rollinger, J.M.; Heiss, E.H.; et al. Natural product agonists of peroxisome proliferator-activated receptor gamma (PPAR $\gamma$ ): A review. Biochem. Pharmacol. 2014, 92, 73-89. [CrossRef]

33. Luo, R.; Fang, D.; Chu, P.; Wu, H.; Zhang, Z.; Tang, Z. Multiple molecular targets in breast cancer therapy by betulinic acid. Biomed. Pharmacother. 2016, 84, 1321-1330. [CrossRef]

34. Synthesis of 3,3,3-trifluoroethyl Isocyanate, Carbamate and Ureas. Anticancer Activity Evaluation of N-(3,3,3-trifluoroethyl)-N'-Substituted Ureas. Available online: http:/ /www.pharmaexpert.ru/passonline/ index.php (accessed on 10 September 2018).

35. Chin, Y.R.; Toker, A. Function of Akt/PKB signaling to cell motility, invasion and the tumor stroma in cancer. Cell. Signal. 2009, 21, 470-476. [CrossRef]

36. Feng, L.X.; Sun, P.; Mi, T.; Liu, M.; Liu, W.; Yao, S.; Cao, Y.M.; Yu, X.L.; Wu, W.Y.; Jiang, B.H.; et al. Agglutinin isolated from Arisema heterophyllum Blume induces apoptosis and autophagy in A549 cells through inhibiting PI3K/Akt pathway and inducing ER stress. Chin. J. Nat. Med. 2016, 14, 856-864. [CrossRef]

37. Jabbarzadeh Kaboli, P.; Pui-Yee Leong, M.; Ismail, P.; Ling, K.H. Antitumor effects of berberine against EGFR, ERK1/2, P38 and AKT in MDA-MB231 and MCF-7 breast cancer cells using molecular modelling and in vitro study. Pharmacol. Rep. 2019, 71, 13-23. [CrossRef]

38. Wietrzyk, J.; Chodyński, M.; Fitak, H.; Wojdat, E.; Kutner, A.; Opolski, A. Antitumor properties of diastereomeric and geometric analogs of vitamin D3. Anticancer Drugs 2007, 18, 447-457. [CrossRef] 
39. ACD Labs. Available online: https:/ / www.acdlabs.com (accessed on 22 November 2018).

40. Frisch, M.J.; Trucks, G.W.; Schlegel, H.B.; Scuseria, G.E.; Robb, M.A.; Cheeseman, J.R.; Scalmani, G.; Barone, V.; Petersson, G.A.; Nakatsuji, H.; et al. Gaussian 16; Revision A; Gaussian Inc.: Wallingford, CT, USA, 2016.

41. Stephens, P.J.; Devlin, F.J.; Chablowski, C.F.; Frisch, M. Ab initio calculation of vibrational absorption and circular dichroism spectra using density functional force fields. J. Phys. Chem. 1994, 98, 11623-11627. [CrossRef]

42. Cole, J.C.; Nissink, J.W.M.; Taylor, R. Protein-ligand Docking and Virtual Screening with GOLD in Virtual Screening in Drug Discovery; Shoichet, B., Alvarez, J., Eds.; Taylor \& Francis CRC Press: Boca Raton, FL, USA, 2005.

43. Dessault Systemes. BIOVIA, Discovery Studio Modeling Environment; Release 2017; De-Ssault Systemes: San Diego, CA, USA, 2016.

(C) 2019 by the authors. Licensee MDPI, Basel, Switzerland. This article is an open access article distributed under the terms and conditions of the Creative Commons Attribution (CC BY) license (http://creativecommons.org/licenses/by/4.0/). 